\title{
Long-Range Exciton-Coupled Circular Dichroism: Application for Determination of Absolute Configuration of Oligonaphthalenes.
}

\author{
Kazunori Tsubaki*, Kazuto Takaishi, Hiroyuki Tanaka, Masaya Miura, Takeo Kawabata \\ Institute for Chemical Research, Kyoto University, Gokasho, Uji, Kyoto, 611-0011, Japan. \\ Email tsubaki@fos.kuicr.kyoto-u.ac.jp
}

Pages 2-8: $\quad$ Synthesis of the Oligonaphthalene Derivatives.

Pages 9-22: $\quad{ }^{1} \mathrm{H}-\mathrm{NMR}$ charts for compounds 1a-7a and 9a-11a.

Pages 23-37: $\quad{ }^{1} \mathrm{H}-\mathrm{NMR}$ charts for compounds $\mathbf{1 b - 7 \mathbf { b }}$ and $\mathbf{9 b - 1 1 b .}$

Page 38: $\quad$ UV-vis and CD spectra of 9b-11b.

Page 39: $\quad$ X-ray structures of ternaphthalenes.

Page 40: $\quad$ X-ray structure of quaternaphthalene.

Pages 41-42: $\quad$ The lowest-energy structures of octinaphthalenes (calculated with CONFLEX-MM2).

Page 43: $\quad$ The lowest-energy structures of $\mathbf{1 b}$ (calculated withCONFLEX-MM2)

Pages 44-46: $\quad$ Original (before smoothing) CD spectra of $\mathbf{1 b}-\mathbf{7 b}$ and $\mathbf{9 b - 1 1 b .}$ 
Scheme S-1. Synthesis of Bisporphyrin Derivatives 1b-7b and 9b-11b.

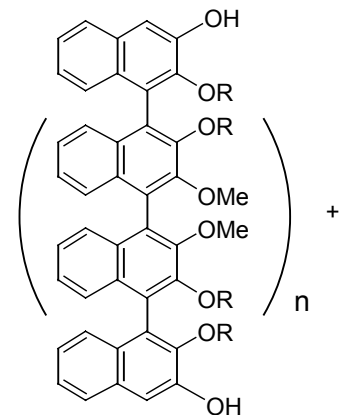

1a: $n=0, R=M e$

2a: $n=1, R=M e$

3a: $n=3, R=M e$

4a: $n=7, R=M e$

5a: $n=0, R=\mathrm{CH}_{2} \mathrm{CON}(E t)_{2}$

6a: $n=1, R=\mathrm{CH}_{2} \mathrm{CON}(\mathrm{Et})_{2}$

7a: $n=3, \mathrm{R}=\mathrm{CH}_{2} \mathrm{CON}(\mathrm{Et})_{2}$

9a: $\mathrm{n}=0, \mathrm{R}=n-\mathrm{Bu}$

10a: $\mathrm{n}=1, \mathrm{R}=n-\mathrm{Bu}$

11a: $\mathrm{n}=3, \mathrm{R}=n-\mathrm{Bu}$

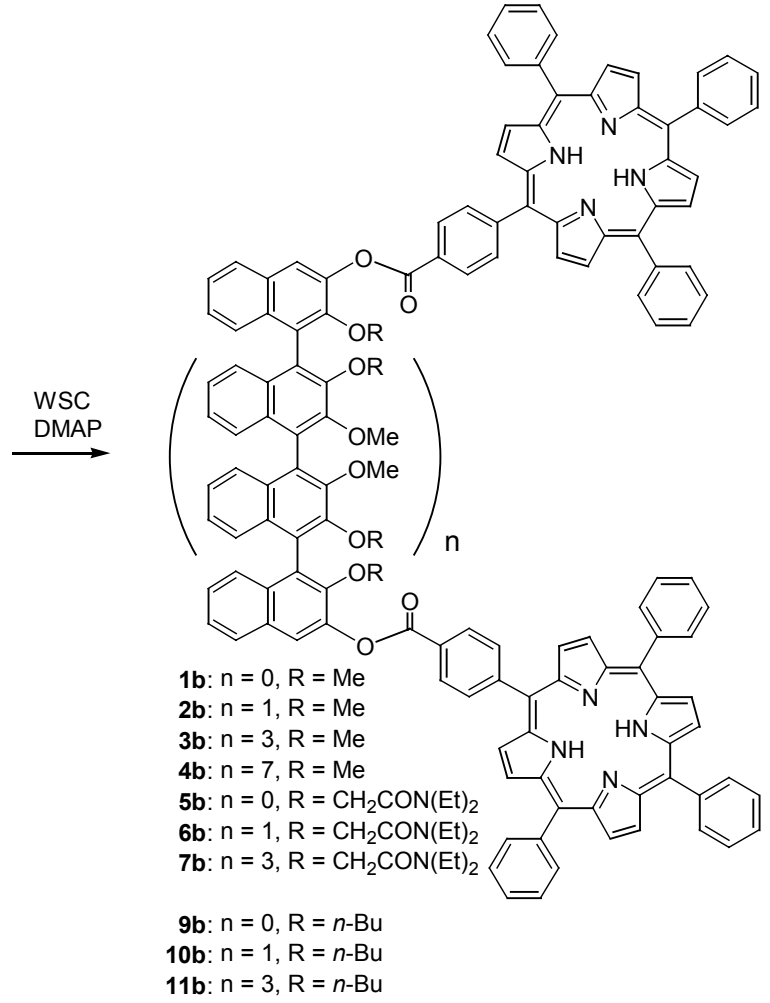


General Procedure for the Synthesis of Diol Compounds 1a-7a and 9a-11a: The synthesis of $(R, R, R)-5 \mathbf{a}$ is typical. To a suspension of dibenzyl ether of $(R, R, R)-5 \mathbf{a}$ (76 mg, $0.058 \mathrm{mmol})$ and $10 \% \mathrm{Pd} / \mathrm{C}(20 \mathrm{mg})$ in THF $(5 \mathrm{ml})$ was stirred at room temperature for 25 hours under $\mathrm{H}_{2}$ balloon. The $\mathrm{Pd} / \mathrm{C}$ was filtered off and evaporated in vacuo to give a residue. The residue was purified by PTLC $\left(\mathrm{SiO}_{2}, n\right.$-hexane/ethyl acetate $\left.=2 / 1\right)$ to afford $(R, R, R)-5 a(38 \mathrm{mg}, 59 \%)$.

General Procedure for the Synthesis of Bisporphyrin Compounds $1 \mathrm{~b}-7 \mathrm{~b}$ and 9b-11b: The synthesis of $(S)-\mathbf{1 b}$ is typical. To a solution of $\mathbf{1 a}(10 \mathrm{mg}, 0.029 \mathrm{mmol})$ in $\mathrm{CH}_{2} \mathrm{Cl}_{2}(3 \mathrm{ml})$, TPP- $\mathrm{CO}_{2} \mathrm{H}(42 \mathrm{mg}, 0.064 \mathrm{mmol}), \mathrm{WSC} \cdot \mathrm{HCl}(111 \mathrm{mg}, 0.58 \mathrm{mmol})$ and DMAP (21 mg, $0.17 \mathrm{mmol})$ were added under argon atmosphere and stirred at room temperature for $24 \mathrm{hrs}$. The reaction mixture was quenched with water, extracted with EtOAc, washed successively with water and brine, dried over $\mathrm{Na}_{2} \mathrm{SO}_{4}$ and evaporated to give a residue which was purified by recycling preparative HPLC (Japan Analytical Industry Co., Ltd. LC-908) with connected JAIGEL-1H (20 x $600 \mathrm{~mm})$ and JAIGEL-2H $(20 \times 600 \mathrm{~mm})$ under the conditions of $3.5 \mathrm{ml} / \mathrm{min}$ of flow rate with $\mathrm{CHCl}_{3}$ detected by UV $(254 \mathrm{~nm})$ and RI (refractive index) to afford $(S)-\mathbf{1 b}$.

(S)-1a: pale yellow foam; $[\alpha]_{\mathrm{D}}{ }^{19}=+149\left(\mathrm{c}=1.00, \mathrm{CHCl}_{3}\right)$; IR $(\mathrm{KBr}) 3411,1507,1466$, $1428 \mathrm{~cm}^{-1}$; ${ }^{1} \mathrm{H}-\mathrm{NMR}\left(200 \mathrm{MHz}, \mathrm{CDCl}_{3}\right) \delta 3.33(\mathrm{~s}, 6 \mathrm{H}), 6.16(\mathrm{~s}, 2 \mathrm{H}), 7.10-7.15(\mathrm{~m}, 4 \mathrm{H})$, 7.30-7.40 (m, 2H), $7.48(\mathrm{~s}, 2 \mathrm{H}), 7.77(\mathrm{~d}, J=8.4,2 \mathrm{H})$; HRMS $\left(\mathrm{EI}^{+}\right)$Calcd for $\mathrm{C}_{22} \mathrm{H}_{18} \mathrm{O}_{4}$ : 346.1205. Found: 346.1208; Anal. Calcd for $\mathrm{C}_{22} \mathrm{H}_{18} \mathrm{O}_{4}$ : C, 76.29; H, 5.24. Found: C, 75.90; $\mathrm{H}, 5.28$.

$(S, S, S)-2 \mathbf{a}: \mathrm{mp}=146{ }^{\circ} \mathrm{C} ;[\alpha]_{\mathrm{D}}{ }^{19}=-85\left(\mathrm{c}=1.00, \mathrm{CHCl}_{3}\right) ; \mathrm{IR}(\mathrm{KBr}) 3501,1507,1451,1242$ $\mathrm{cm}^{-1} ;{ }^{1} \mathrm{H}-\mathrm{NMR}\left(200 \mathrm{MHz}, \mathrm{CDCl}_{3}\right) \delta 3.61(\mathrm{~s}, 6 \mathrm{H}), 3.74(\mathrm{~s}, 6 \mathrm{H}), 3.79(\mathrm{~s}, 6 \mathrm{H}), 6.22(\mathrm{~s}, 2 \mathrm{H})$, 7.15-7.55 (m, 14H), $7.52(\mathrm{~s}, 2 \mathrm{H}), 7.82(\mathrm{~d}, J=8.4,2 \mathrm{H})$; HRMS $\left(\mathrm{EI}^{+}\right)$Calcd for $\mathrm{C}_{46} \mathrm{H}_{38} \mathrm{O}_{8}$ : 718.2566. Found: 718.2585; Anal. Calcd for $\mathrm{C}_{46} \mathrm{H}_{38} \mathrm{O}_{8} \cdot 0.6 \mathrm{CHCl}_{3}: \mathrm{C}, 70.81 ; \mathrm{H}, 4.92$. Found: C, $70.75 ; \mathrm{H}, 5.02$.

$(S, R, S)-\mathbf{2 a}: \mathrm{mp}=161^{\circ} \mathrm{C} ;[\alpha]_{\mathrm{D}}{ }^{20}=-50\left(\mathrm{c}=0.15, \mathrm{CHCl}_{3}\right) ; \mathrm{IR}(\mathrm{KBr}) 3503,1501,1450,1239$ 
$\mathrm{cm}^{-1} ;{ }^{1} \mathrm{H}-\mathrm{NMR}\left(200 \mathrm{MHz}, \mathrm{CDCl}_{3}\right) \delta 3.50(\mathrm{~s}, 6 \mathrm{H}), 3.73(\mathrm{~s}, 12 \mathrm{H}), 6.21(\mathrm{~s}, 2 \mathrm{H}), 7.18-7.51(\mathrm{~m}$, $14 \mathrm{H}), 7.52(\mathrm{~s}, 2 \mathrm{H}), 7.83(\mathrm{~d}, J=8.6,2 \mathrm{H})$; HRMS $\left(\mathrm{EI}^{+}\right)$Calcd for $\mathrm{C}_{46} \mathrm{H}_{38} \mathrm{O}_{8}: 718.2567$. Found: 718.2573.

(S,S,S,S,S,S,S)-3a: known; $\quad$ Furuta, T.; Tanaka, K.; Tsubaki, K.; Fuji, K.

Tetrahedron, 2004, 60, 4431-4441.

(S,S,S,R,S,S,S)-3a: known; $\quad$ Furuta, T.; Tanaka, K.; Tsubaki, K.; Fuji, K.

Tetrahedron, 2004, 60, 4431-4441.

$(S, S, S, S, S, S, S, S, S, S, S, S, S, S, S)-\mathbf{4 a}: \mathrm{mp}>300^{\circ} \mathrm{C} ;[\alpha]_{\mathrm{D}}{ }^{19}=-214\left(\mathrm{c}=0.50, \mathrm{CHCl}_{3}\right) ; \mathrm{IR}(\mathrm{KBr})$ 3648, 2933, 1507, $1237 \mathrm{~cm}^{-1}$; ${ }^{1} \mathrm{H}-\mathrm{NMR}\left(200 \mathrm{MHz}, \mathrm{CDCl}_{3}\right) \delta 3.65(\mathrm{~s}, 6 \mathrm{H}), 3.76(\mathrm{~s}, 6 \mathrm{H})$, $3.80(\mathrm{~s}, 6 \mathrm{H}), 3.96$ (brs, 72H), $6.23(\mathrm{~s}, 2 \mathrm{H}), 7.23-7.55(\mathrm{~m}, 64 \mathrm{H}), 7.83(\mathrm{~d}, J=7.9,2 \mathrm{H})$; MALDI-TOF-MS (dithranol) 2952.86

$(S, S, S, S, S, S, S, R, S, S, S, S, S, S, S)-\mathbf{4 a}: \mathrm{mp}>300{ }^{\circ} \mathrm{C} ;[\alpha]_{\mathrm{D}}{ }^{19}=-202\left(\mathrm{c}=0.25, \mathrm{CHCl}_{3}\right) ; \mathrm{IR}(\mathrm{KBr})$ 2938, 1507, 1457, $1387 \mathrm{~cm}^{-1}$; ${ }^{1} \mathrm{H}-\mathrm{NMR}\left(200 \mathrm{MHz}, \mathrm{CDCl}_{3}\right) \delta 3.65(\mathrm{~s}, 6 \mathrm{H}), 3.76(\mathrm{~s}, 6 \mathrm{H})$, $3.83(\mathrm{~s}, 6 \mathrm{H}), 3.87(\mathrm{~s}, 6 \mathrm{H}), 3.89(\mathrm{~s}, 6 \mathrm{H}), 3.90-4.05(\mathrm{~m}, 60 \mathrm{H}), 6.24(\mathrm{~s}, 2 \mathrm{H}), 7.23-7.60(\mathrm{~m}$, 64H), 7.84 (d, $J=7.6,2 \mathrm{H}$ ); MALDI-TOF-MS (dithranol) 2952.44.

(R)-5a: $\mathrm{mp}=170{ }^{\circ} \mathrm{C} ;[\alpha]_{\mathrm{D}}{ }^{20}=-92\left(\mathrm{c}=0.10, \mathrm{CHCl}_{3}\right)$; IR $(\mathrm{KBr}) 2979,1640,1511,1444$ $\mathrm{cm}^{-1} ;{ }^{1} \mathrm{H}-\mathrm{NMR}\left(200 \mathrm{MHz}, \mathrm{CDCl}_{3}\right) \delta 0.76(\mathrm{t}, J=6.8,6 \mathrm{H}), 1.06(\mathrm{t}, J=6.8,6 \mathrm{H}), 2.66(\mathrm{q}, J=$ $7.2,4 \mathrm{H}), 3.10-3.50(\mathrm{~m}, 4 \mathrm{H}), 4.04(\mathrm{~d}, J=15.8,2 \mathrm{H}), 4.22(\mathrm{~d}, J=15.8,2 \mathrm{H}), 7.0-7.15(\mathrm{~m}, 4 \mathrm{H})$, 7.3-7.4 (m, 2H), 7.49 (s, 2H), $7.77(\mathrm{~d}, J=8.0,2 \mathrm{H}), 10.79$ (brs, 2H); HRMS (EI $\left.{ }^{+}\right)$Calcd for $\mathrm{C}_{32} \mathrm{H}_{36} \mathrm{O}_{6} \mathrm{~N}_{2}:$ 544.2573. Found: 544.2569.

$(R, R, R)$-6a: $\mathrm{mp}=180{ }^{\circ} \mathrm{C} ;[\alpha]_{\mathrm{D}}^{21}=+84\left(\mathrm{c}=0.61, \mathrm{CHCl}_{3}\right) ; \mathrm{IR}(\mathrm{KBr}) 2974,1641,1549$, $1443 \mathrm{~cm}^{-1} ;{ }^{1} \mathrm{H}-\mathrm{NMR}\left(200 \mathrm{MHz}, \mathrm{CDCl}_{3} / \mathrm{CD}_{3} \mathrm{OD}=1 / 1\right) \delta 0.57(\mathrm{t}, J=7.0,6 \mathrm{H}), 0.90(\mathrm{t}, J=$ $7.2,6 \mathrm{H}), 0.94(\mathrm{t}, J=7.8,6 \mathrm{H}), 1.10(\mathrm{t}, J=7.2,6 \mathrm{H}), 2.45-2.65(\mathrm{~m}, 4 \mathrm{H}), 2.8-3.1(\mathrm{~m}, 4 \mathrm{H})$, $3.14(\mathrm{q}, J=7.0,4 \mathrm{H}), 3.37(\mathrm{q}, J=7.2,4 \mathrm{H}), 3.84(\mathrm{~s}, 6 \mathrm{H}), 4.31(\mathrm{~d}, J=12.0,2 \mathrm{H}), 4.52(\mathrm{~d}, J=$ 15.6, 2H), 4.67 (d, $J=15.6,2 \mathrm{H}), 4.72(\mathrm{~d}, J=12.0,2 \mathrm{H}), 7.1-7.5(\mathrm{~m}, 14 \mathrm{H}), 7.53(\mathrm{~s}, 2 \mathrm{H})$, $7.80(\mathrm{~d}, J=8.4,2 \mathrm{H})$; HRMS $\left(\mathrm{FAB}^{+}\right.$) Calcd for $\mathrm{C}_{66} \mathrm{H}_{75} \mathrm{O}_{12} \mathrm{~N}_{4}$ : 1115.5382. Found: 1115.5371 . Anal. Calcd for $\mathrm{C}_{66} \mathrm{H}_{74} \mathrm{O}_{12} \mathrm{~N}_{4} \cdot 1.3 \mathrm{CHCl}_{3}$ : C, 63.62; H, 5.97; N, 4.41. Found: C, 63.86; H, $5.94 ; \mathrm{N}, 4.33$. 
$(R, R, R, R, R, R, R)-7 \mathbf{a}: \mathrm{mp}=132{ }^{\circ} \mathrm{C} ;[\alpha]_{\mathrm{D}}{ }^{20}=+174\left(\mathrm{c}=0.50, \mathrm{CHCl}_{3}\right) ; \mathrm{IR}(\mathrm{KBr}) 3465,2972$, 1649, $1452 \mathrm{~cm}^{-1}$; ${ }^{1} \mathrm{H}-\mathrm{NMR}\left(200 \mathrm{MHz}, \mathrm{CDCl}_{3}\right) \delta 0.58(\mathrm{t}, J=6.8,6 \mathrm{H}), 0.65-1.3(\mathrm{~m}, 42 \mathrm{H})$, 2.55-2.65 (m, 8H), 2.8-3.6 (m, 24H), $3.87(\mathrm{~s}, 6 \mathrm{H}), 3.92(\mathrm{~s}, 6 \mathrm{H}), 3.94(\mathrm{~s}, 6 \mathrm{H}), 4.2-4.8(\mathrm{~m}$, 12H), $4.99(\mathrm{~d}, J=12.2,4 \mathrm{H}), 7.2-7.6(\mathrm{~m}, 32 \mathrm{H}), 7.82(\mathrm{~d}, J=8.2,2 \mathrm{H}), 10.85(\mathrm{~s}, 2 \mathrm{H}) ; \mathrm{MS}$ $\left(\mathrm{FAB}^{+}\right) 2278\left(\mathrm{M}+\mathrm{Na}^{+}\right)$. Anal. Calcd for $\mathrm{C}_{134} \mathrm{H}_{150} \mathrm{O}_{24} \mathrm{~N}_{8} \cdot 3 \mathrm{CHCl}_{3}$ : C, 62.93; H, 5.90; N, 4.29. Found: C, 63.24; H, 6.21; N, 3.87 .

(S)-9a: $\mathrm{mp}=76{ }^{\circ} \mathrm{C} ;[\alpha]_{\mathrm{D}}{ }^{22}=+201\left(\mathrm{c}=0.12, \mathrm{CHCl}_{3}\right) ; \mathrm{IR}(\mathrm{KBr}) 3417,2959,1622,1598$ $\mathrm{cm}^{-1} ;{ }^{1} \mathrm{H}-\mathrm{NMR}\left(200 \mathrm{MHz}, \mathrm{CDCl}_{3}\right) \delta 0.57(\mathrm{t}, J=7.2,6 \mathrm{H}), 0.8-1.1(\mathrm{~m}, 4 \mathrm{H}), 1.2-1.4(\mathrm{~m}, 4 \mathrm{H})$, 3.25-3.55 (m, 4H), $6.18(\mathrm{~s}, 2 \mathrm{H}), 7.05-7.20(\mathrm{~m}, 4 \mathrm{H}), 7.3-7.4(\mathrm{~m}, 2 \mathrm{H}), 7.46(\mathrm{~s}, 2 \mathrm{H}), 7.76(\mathrm{~d}, J$ $=8.4,2 \mathrm{H}$ ); HRMS $\left(\mathrm{EI}^{+}\right)$Calcd for $\mathrm{C}_{28} \mathrm{H}_{30} \mathrm{O}_{4}: 430.2144$. Found: 430.2158; Anal. Calcd for $\mathrm{C}_{28} \mathrm{H}_{30} \mathrm{O}_{4}: \mathrm{C}, 78.11 ; \mathrm{H}, 7.02$. Found: $\mathrm{C}, 78.20 ; \mathrm{H}, 7.11$.

$(S, S, S)-10 a$ : colorless foam; $[\alpha]_{\mathrm{D}}^{20}=-77\left(\mathrm{c}=0.10, \mathrm{CHCl}_{3}\right)$; IR $\left(\mathrm{CDCl}_{3}\right) 3518,2960,1508$, $1442 \mathrm{~cm}^{-1} ;{ }^{1} \mathrm{H}-\mathrm{NMR}\left(200 \mathrm{MHz}, \mathrm{CDCl}_{3}\right) \delta 0.53(\mathrm{t}, J=7.2,6 \mathrm{H}), 0.75(\mathrm{t}, J=7.2,6 \mathrm{H}), 0.8-1.6$ (m, 16H), 3.55-3.85 (m, 4H), 3.77 (s, 6H), 4.00-4.20 (m, 4H), 6.19 (s, 2H), 7.10-7.45 (m, 14H), $7.51(\mathrm{~s}, 2 \mathrm{H}), 7.81(\mathrm{~d}, J=8.4,2 \mathrm{H})$; HRMS $\left(\mathrm{FAB}^{+}\right)$Calcd for $\mathrm{C}_{58} \mathrm{H}_{62} \mathrm{O}_{8}: 886.4445$. Found: 886.4450; Anal. Calcd for $\mathrm{C}_{58} \mathrm{H}_{62} \mathrm{O}_{8}$ : C, 78.53; H, 7.04. Found: $\mathrm{C}, 78.31 ; \mathrm{H}, 7.10$.

$(S, R, S)-10 a$ : colorless foam; $[\alpha]_{\mathrm{D}}^{20}=-65\left(\mathrm{c}=0.10, \mathrm{CHCl}_{3}\right)$; IR $(\mathrm{KBr}) 3508,2957,1442$, $1376 \mathrm{~cm}^{-1} ;{ }^{1} \mathrm{H}-\mathrm{NMR}\left(200 \mathrm{MHz}, \mathrm{CDCl}_{3}\right) \delta 0.55(\mathrm{t}, J=7.2,6 \mathrm{H}), 0.71(\mathrm{t}, J=7.2,6 \mathrm{H})$, 0.75-1.60 (m, 16H), 3.50-3.85 (m, 6H), $3.72(\mathrm{~s}, 6 \mathrm{H}), 3.95-4.10(\mathrm{~m}, 2 \mathrm{H}), 6.22(\mathrm{~s}, 2 \mathrm{H})$, 7.10-7.45 (m, 14H), $7.50(\mathrm{~s}, 2 \mathrm{H}), 7.81(\mathrm{~d}, J=8.0,2 \mathrm{H})$; HRMS $\left(\mathrm{FAB}^{+}\right)$Calcd for $\mathrm{C}_{58} \mathrm{H}_{62} \mathrm{O}_{8}$ : 886.4445. Found: 886.4418; Anal. Calcd for $\mathrm{C}_{58} \mathrm{H}_{62} \mathrm{O}_{8}$ : C, 78.53; H, 7.04. Found: C, 78.82; $\mathrm{H}, 7.17$

$(S, S, S, S, S, S, S)-11 \mathrm{a}:$ foam; $[\alpha]_{\mathrm{D}}{ }^{22}=-159\left(\mathrm{c}=0.12, \mathrm{CHCl}_{3}\right)$; IR $(\mathrm{KBr}) 2957,1506,1444$, $1398 \mathrm{~cm}^{-1}$; ${ }^{1} \mathrm{H}-\mathrm{NMR}\left(200 \mathrm{MHz}, \mathrm{CDCl}_{3}\right) \delta 0.54(\mathrm{t}, J=7.0,6 \mathrm{H}), 0.65-0.85(\mathrm{~m}, 18 \mathrm{H}), 0.8-1.6$ $(\mathrm{m}, 32 \mathrm{H}), 3.6-4.4(\mathrm{~m}, 16 \mathrm{H}), 3.81(\mathrm{~s}, 6 \mathrm{H}), 3.88(\mathrm{~s}, 6 \mathrm{H}), 3.90(\mathrm{~s}, 6 \mathrm{H}), 6.21(\mathrm{~s}, 2 \mathrm{H}), 7.1-7.5(\mathrm{~m}$, 32H), $7.52(\mathrm{~s}, 2 \mathrm{H}), 7.82(\mathrm{~d}, J=8.2,2 \mathrm{H})$; MS $\left(\mathrm{FAB}^{+}\right) 1977\left(\mathrm{M}^{+}\right)$. Anal. Calcd for $\mathrm{C}_{118} \mathrm{H}_{126} \mathrm{O}_{16}$ : C, 78.73; H, 7.05. Found: C, 78.76; H, 7.16. 
(S)-1b: $76 \%$ yield; $\mathrm{mp}>300{ }^{\circ} \mathrm{C} ;[\alpha]_{\mathrm{D}}{ }^{20}=-1380\left(\mathrm{c}=0.10, \mathrm{CHCl}_{3}\right)$; IR $(\mathrm{KBr}) 3053,1741$, 1600, $1469 \mathrm{~cm}^{-1} ;{ }^{1} \mathrm{H}-\mathrm{NMR}\left(200 \mathrm{MHz}, \mathrm{CDCl}_{3}\right) \delta-2.76(\mathrm{~s}, 4 \mathrm{H}), 3.75(\mathrm{~s}, 6 \mathrm{H}), 7.21-7.75(\mathrm{~m}$, 24H), 7.99 (d, $J=7.8,2 \mathrm{H}), 8.08(\mathrm{~s}, 2 \mathrm{H}), 8.17-8.25(\mathrm{~m}, 12 \mathrm{H}), 8.41$ (d, $J=7.0,4 \mathrm{H}), 8.70$ (d, $J=7.0,4 \mathrm{H}), 8.81-8.92(\mathrm{~m}, 16 \mathrm{H})$; HRMS $\left(\mathrm{FAB}^{+}\right)$Calcd for $\mathrm{C}_{112} \mathrm{H}_{75} \mathrm{O}_{6} \mathrm{~N}_{8}$ : 1627.5810 . Found: 1627.5795; Anal. Calcd for $\mathrm{C}_{112} \mathrm{H}_{74} \mathrm{O}_{6} \mathrm{~N}_{8} \cdot 1.1 \mathrm{CHCl}_{3}$ : C, 77.22; H, 4.30; N, 6.37. Found: C, 77.18; H, 4.46; N, 6.35 .

$(S, S, S)-\mathbf{2 b}: 53 \%$ yield; $\mathrm{mp}>300{ }^{\circ} \mathrm{C} ;[\alpha]_{\mathrm{D}}{ }^{20}=-2030\left(\mathrm{c}=0.070, \mathrm{CHCl}_{3}\right) ; \mathrm{IR}(\mathrm{KBr}) 2938$, 1741, 1602, $1558 \mathrm{~cm}^{-1} ;{ }^{1} \mathrm{H}-\mathrm{NMR}\left(200 \mathrm{MHz}, \mathrm{CDCl}_{3}\right) \delta-2.76(\mathrm{~s}, 4 \mathrm{H}), 3.83(\mathrm{~s}, 6 \mathrm{H}), 3.87(\mathrm{~s}$, $6 \mathrm{H}), 3.89(\mathrm{~s}, 6 \mathrm{H}), 7.2-7.6(\mathrm{~m}, 14 \mathrm{H}), 7.6-7.8(\mathrm{~m}, 18 \mathrm{H}), 8.04(\mathrm{~d}, J=8.2,2 \mathrm{H}), 8.10(\mathrm{~s}, 2 \mathrm{H})$, 8.1-8.3 (m, 12H), $8.43(\mathrm{~d}, J=7.8,4 \mathrm{H}), 8.73(\mathrm{~d}, J=7.8,4 \mathrm{H}), 8.8-8.95(\mathrm{~m}, 16 \mathrm{H})$; MS $\left(\mathrm{FAB}^{+}\right) 2000\left(\mathrm{M}^{+}\right)$; Anal. Calcd for $\mathrm{C}_{136} \mathrm{H}_{94} \mathrm{O}_{10} \mathrm{~N}_{8} \cdot 1.6 \mathrm{CHCl}_{3}$ : C, 75.42; H, 4.40; N, 5.11 . Found: C, 75.38; H, 4.64; N, 5.21.

$(S, R, S)-2 \mathbf{b}: 80 \%$ yield; $\mathrm{mp}>300{ }^{\circ} \mathrm{C} ;[\alpha]_{\mathrm{D}}{ }^{20}=-1760\left(\mathrm{c}=0.11, \mathrm{CHCl}_{3}\right) ; \mathrm{IR}(\mathrm{KBr}) 2936$, 1741, 1602, $1469 \mathrm{~cm}^{-1} ;{ }^{1} \mathrm{H}-\mathrm{NMR}\left(200 \mathrm{MHz}, \mathrm{CDCl}_{3}\right) \delta-2.76(\mathrm{~s}, 4 \mathrm{H}), 3.74(\mathrm{~s}, 6 \mathrm{H}), 3.79(\mathrm{~s}$, $6 \mathrm{H}), 3.87(\mathrm{~s}, 6 \mathrm{H}), 7.23-7.77(\mathrm{~m}, 32 \mathrm{H}), 8.03(\mathrm{~d}, J=8.4,2 \mathrm{H}), 8.08(\mathrm{~s}, 2 \mathrm{H}), 8.20-8.25(\mathrm{~m}$, $12 \mathrm{H}), 8.42(\mathrm{~d}, J=8.0,4 \mathrm{H}), 8.72(\mathrm{~d}, J=8.0,4 \mathrm{H}), 8.75-8.93(\mathrm{~m}, 16 \mathrm{H}) ; \mathrm{MS}\left(\mathrm{FAB}^{+}\right) 2000$ $\left(\mathrm{M}^{+}\right)$; Anal. Calcd for $\mathrm{C}_{136} \mathrm{H}_{94} \mathrm{O}_{10} \mathrm{~N}_{8} \cdot 1.5 \mathrm{CHCl}_{3}: \mathrm{C}, 75.78 ; \mathrm{H}, 4.42 ; \mathrm{N}, 5.14$. Found: $\mathrm{C}, 75.55$; $\mathrm{H}, 4.66 ; \mathrm{N}, 5.23$.

$(S, S, S, S, S, S, S)-\mathbf{3 b}: 76 \%$ yield; $\mathrm{mp}>300{ }^{\circ} \mathrm{C} ;[\alpha]_{\mathrm{D}}{ }^{20}=-2640\left(\mathrm{c}=0.10, \mathrm{CHCl}_{3}\right)$; IR $(\mathrm{KBr})$ 2936, 1742, 1451, $1346 \mathrm{~cm}^{-1}$; ${ }^{1} \mathrm{H}-\mathrm{NMR}\left(200 \mathrm{MHz}, \mathrm{CDCl}_{3}\right) \delta-2.76(\mathrm{~s}, 4 \mathrm{H}), 3.85-3.98(\mathrm{~m}$, $42 \mathrm{H}), 7.24-7.79(\mathrm{~m}, 48 \mathrm{H}), 8.04(\mathrm{~d}, J=8.4,2 \mathrm{H}), 8.11(\mathrm{~s}, 2 \mathrm{H}), 8.20-8.26(\mathrm{~m}, 12 \mathrm{H}), 8.44(\mathrm{~d}, J$ $=7.6,4 \mathrm{H}), 8.74(\mathrm{~d}, J=7.6,4 \mathrm{H}), 8.85-8.92(\mathrm{~m}, 16 \mathrm{H}) ; \mathrm{MS}_{\left(\mathrm{FAB}^{+}\right)} 2745\left(\mathrm{M}^{+}\right)$; Anal. Calcd for $\mathrm{C}_{184} \mathrm{H}_{134} \mathrm{O}_{18} \mathrm{~N}_{8} \cdot 2.1 \mathrm{CHCl}_{3}: \mathrm{C}, 74.61 ; \mathrm{H}, 4.58 ; \mathrm{N}, 3.74$. Found: C, 74.25; H, 4.93; N, 3.88 .

$(S, S, S, R, S, S, S)-3 \mathbf{b}: 22 \%$ yield; $\mathrm{mp}>300{ }^{\circ} \mathrm{C} ;[\alpha]_{\mathrm{D}}^{20}=-2530\left(\mathrm{c}=0.054, \mathrm{CHCl}_{3}\right) ; \mathrm{IR}(\mathrm{KBr})$ 2936, 1740, 1453, $1386 \mathrm{~cm}^{-1}$; ${ }^{1} \mathrm{H}-\mathrm{NMR}\left(200 \mathrm{MHz}, \mathrm{CDCl}_{3}\right) \delta-2.76(\mathrm{~s}, 4 \mathrm{H}), 3.83-3.98(\mathrm{~m}$, $42 \mathrm{H}), 7.24-7.80(\mathrm{~m}, 48 \mathrm{H}), 8.11-8.25(\mathrm{~m}, 16 \mathrm{H}), 8.44(\mathrm{~d}, J=8.4,4 \mathrm{H}), 8.74(\mathrm{~d}, J=8.4,4 \mathrm{H})$, 8.85-8.93 (m, 16H); MS (FAB $\left.{ }^{+}\right) 2745\left(\mathrm{M}^{+}\right)$. 
$(S, S, S, S, S, S, S, S, S, S, S, S, S, S, S)-\mathbf{4 b}: 67 \%$ yield; $\mathrm{mp}>300{ }^{\circ} \mathrm{C} ;[\alpha]_{\mathrm{D}}^{21}=-3000(\mathrm{c}=0.09$, $\mathrm{CHCl}_{3}$ ); IR (KBr) 2937, 1744, 1451, $1387 \mathrm{~cm}^{-1} ;{ }^{1} \mathrm{H}-\mathrm{NMR}\left(200 \mathrm{MHz}, \mathrm{CDCl}_{3}\right) \delta-2.75(\mathrm{~s}$, $4 \mathrm{H}), 3.6-4.15(\mathrm{~m}, 90 \mathrm{H}), 7.2-7.6(\mathrm{~m}, 66 \mathrm{H}), 7.6-7.9(\mathrm{~m}, 18 \mathrm{H}), 8.0-8.3(\mathrm{~m}, 12 \mathrm{H}), 8.44(\mathrm{~d}, J=$ 7.6, 4H), 8.74 (d, $J=7.6,4 \mathrm{H}), 8.8-9.0$ (m, 16H); MALDI-TOF-MS (dithranol) 4234.35; Anal. Calcd for $\mathrm{C}_{280} \mathrm{H}_{214} \mathrm{O}_{34} \mathrm{~N}_{8} \cdot 2.8 \mathrm{CHCl}_{3}: \mathrm{C}, 74.34 ; \mathrm{H}, 4.78 ; \mathrm{N}, 2.45$. Found: C, 74.14; H, $5.14 ; \mathrm{N}, 2.79$.

$(S, S, S, S, S, S, S, R, S, S, S, S, S, S, S)-\mathbf{4 b}: 72 \%$ yield; $\mathrm{mp}>300{ }^{\circ} \mathrm{C} ;[\alpha]_{\mathrm{D}}{ }^{20}=-2980(\mathrm{c}=0.05$, $\mathrm{CHCl}_{3}$ ); IR (KBr) 2936, 1743, 1452, $1387 \mathrm{~cm}^{-1} ;{ }^{1} \mathrm{H}-\mathrm{NMR}\left(200 \mathrm{MHz}, \mathrm{CDCl}_{3}\right) \delta-2.75(\mathrm{~s}$, $4 \mathrm{H}), 3.7-4.1(\mathrm{~m}, 90 \mathrm{H}), 7.2-7.6(\mathrm{~m}, 66 \mathrm{H}), 7.6-7.8(\mathrm{~m}, 18 \mathrm{H}), 8.1-8.3(\mathrm{~m}, 12 \mathrm{H}), 8.44(\mathrm{~d}, J=$ 7.8, 4H), 8.73 (d, $J=7.8,4 \mathrm{H}), 8.8-9.0(\mathrm{~m}, 16 \mathrm{H})$; MALDI-TOF-MS (dithranol) 4234.25.

(R)-5b: $27 \%$ yield; $\mathrm{mp}>300{ }^{\circ} \mathrm{C} ;[\alpha]_{\mathrm{D}}{ }^{20}=-3460\left(\mathrm{c}=0.06, \mathrm{CHCl}_{3}\right) ; \mathrm{IR}(\mathrm{KBr}) 2934,1735$, 1653, $1472 \mathrm{~cm}^{-1} ;{ }^{1} \mathrm{H}-\mathrm{NMR}\left(200 \mathrm{MHz}, \mathrm{CDCl}_{3}\right) \delta-2.77(\mathrm{~s}, 4 \mathrm{H}), 0.78(\mathrm{t}, J=7.0,6 \mathrm{H}), 0.96(\mathrm{t}$, $J=6.8,6 \mathrm{H}), 2.7-3.2(\mathrm{~m}, 8 \mathrm{H}), 4.44(\mathrm{~d}, J=12.2,2 \mathrm{H}), 4.61(\mathrm{~d}, J=12.2,2 \mathrm{H}), 7.3-7.8(\mathrm{~m}$, $24 \mathrm{H}), 8.00(\mathrm{~d}, J=8.0,2 \mathrm{H}), 8.12(\mathrm{~s}, 2 \mathrm{H}), 8.1-8.3(\mathrm{~m}, 12 \mathrm{H}), 8.41(\mathrm{~d}, J=8.2,4 \mathrm{H}), 8.70(\mathrm{~d}, J$ $=8.2,4 \mathrm{H}), 8.80-8.90(\mathrm{~m}, 16 \mathrm{H})$; $\mathrm{MS}\left(\mathrm{FAB}^{+}\right) 1848\left(\mathrm{M}+\mathrm{Na}^{+}\right)$.

$(R, R, R)-6 \mathbf{b}: 40 \%$ yield; $\mathrm{mp}=253{ }^{\circ} \mathrm{C} ;[\alpha]_{\mathrm{D}}{ }^{20}=-3480\left(\mathrm{c}=0.06, \mathrm{CHCl}_{3}\right) ; \mathrm{IR}(\mathrm{KBr}) 2925$, $1741,1648,1604 \mathrm{~cm}^{-1} ;{ }^{1} \mathrm{H}-\mathrm{NMR}\left(200 \mathrm{MHz}, \mathrm{CDCl}_{3}\right) \delta-2.76(\mathrm{~s}, 4 \mathrm{H}), 0.69(\mathrm{t}, J=6.8,6 \mathrm{H})$, 0.8-1.2 (m, 18H), 2.7-3.4 (m, 16H), $3.90(\mathrm{~s}, 6 \mathrm{H}), 4.46(\mathrm{~d}, J=11.6,2 \mathrm{H}), 4.53(\mathrm{~d}, J=13.8$, $2 \mathrm{H}), 4.74(\mathrm{~d}, J=13.8,2 \mathrm{H}), 4.89(\mathrm{~d}, J=11.6,2 \mathrm{H}), 7.2-7.8(\mathrm{~m}, 32 \mathrm{H}), 8.02(\mathrm{~d}, J=7.8,2 \mathrm{H})$, $8.11(\mathrm{~s}, 2 \mathrm{H}), 8.1-8.3(\mathrm{~m}, 12 \mathrm{H}), 8.42(\mathrm{~d}, J=7.6,4 \mathrm{H}), 8.71(\mathrm{~d}, J=7.6,4 \mathrm{H}), 8.8-9.0(\mathrm{~m}, 16 \mathrm{H})$; MS $\left(\mathrm{FAB}^{+}\right) 2418\left(\mathrm{M}+\mathrm{Na}^{+}\right)$.

$(R, R, R, R, R, R, R)-7 \mathbf{b}: 76 \%$ yield; $\mathrm{mp}=215{ }^{\circ} \mathrm{C} ;[\alpha]_{\mathrm{D}}^{20}=-3570\left(\mathrm{c}=0.06, \mathrm{CHCl}_{3}\right) ; \mathrm{IR}(\mathrm{KBr})$ 2935, 1740, 1643, $1442 \mathrm{~cm}^{-1}$; ${ }^{1} \mathrm{H}-\mathrm{NMR}\left(200 \mathrm{MHz}, \mathrm{CDCl}_{3}\right) \delta-2.74(\mathrm{~s}, 4 \mathrm{H}), 0.6-1.3(\mathrm{~m}$, $48 \mathrm{H}), 2.8-3.45(\mathrm{~m}, 32 \mathrm{H}), 3.85-3.95(\mathrm{~m}, 18 \mathrm{H}), 4.4-5.1(\mathrm{~m}, 16 \mathrm{H}), 7.2-7.9(\mathrm{~m}, 48 \mathrm{H}), 8.0-8.4$ (m, 16H), $8.46(\mathrm{~d}, J=6.2,4 \mathrm{H}), 8.73(\mathrm{~d}, J=6.2,4 \mathrm{H}), 8.8-9.0(\mathrm{~m}, 16 \mathrm{H})$; MALDI-TOF-MS (dithranol) 3537.26 .

(S)-9b: $82 \%$ yield; $\mathrm{mp}=294-295{ }^{\circ} \mathrm{C} ;[\alpha]_{\mathrm{D}}{ }^{21}=-3850\left(\mathrm{c}=0.05, \mathrm{CHCl}_{3}\right)$; IR $(\mathrm{KBr}) 1735$, 
1439, $1261 \mathrm{~cm}^{-1} ;{ }^{1} \mathrm{H}-\mathrm{NMR}\left(200 \mathrm{MHz}, \mathrm{CDCl}_{3}\right) \delta-2.77(\mathrm{~s}, 4 \mathrm{H}), 0.63(\mathrm{t}, J=7.4,6 \mathrm{H}), 0.9-1.5$ $(\mathrm{m}, 8 \mathrm{H}), 3.7-4.2(\mathrm{~m}, 4 \mathrm{H}), 7.34-7.58(\mathrm{~m}, 6 \mathrm{H}), 7.62-7.84(\mathrm{~m}, 18 \mathrm{H}), 7.99(\mathrm{~d}, J=8.4,2 \mathrm{H}), 8.06$ (s, 2H), 8.10-8.25 (m, 12H), $8.41(\mathrm{~d}, J=8.0,4 \mathrm{H}), 8.70(\mathrm{~d}, J=8.2,4 \mathrm{H}), 8.80-8.95$ (m, $16 \mathrm{H})$, ; MS $\left(\mathrm{FAB}^{+}\right) 1711\left(\mathrm{M}^{+}\right)$; Anal. Calcd for $\mathrm{C}_{118} \mathrm{H}_{86} \mathrm{O}_{16} \mathrm{~N}_{8} \cdot 1.5 \mathrm{H}_{2} \mathrm{O}: \mathrm{C}, 81.50 ; \mathrm{H}, 5.16 ; \mathrm{N}$, 6.44. Found: C, 81.53; H, 5.07; N, 6.45.

$(S, S, S)-10 b: 81 \%$ yield; $\mathrm{mp}=281-284^{\circ} \mathrm{C} ;[\alpha]_{\mathrm{D}}{ }^{21}=-3360\left(\mathrm{c}=0.05, \mathrm{CHCl}_{3}\right) ; \mathrm{IR}(\mathrm{KBr}) 2956$, 1734, $1243 \mathrm{~cm}^{-1} ;{ }^{1} \mathrm{H}-\mathrm{NMR}\left(300 \mathrm{MHz}, \mathrm{CDCl}_{3}\right) \delta-2.75(\mathrm{~s}, 4 \mathrm{H}), 0.68(\mathrm{t}, J=7.2,6 \mathrm{H}), 0.74(\mathrm{t}$, $J=7.2,6 \mathrm{H}), 0.9-1.3(\mathrm{~m}, 8 \mathrm{H}), 1.3-1.6(\mathrm{~m}, 8 \mathrm{H}), 3.7-3.9(\mathrm{~m}, 4 \mathrm{H}), 3.86(\mathrm{~s}, 6 \mathrm{H}), 4.10-4.25(\mathrm{~m}$, $4 \mathrm{H}), 7.15-7.85(\mathrm{~m}, 32 \mathrm{H}), 8.01(\mathrm{~d}, J=8.1,2 \mathrm{H}), 8.08(\mathrm{~s}, 2 \mathrm{H}), 8.17-8.28(\mathrm{~m}, 12 \mathrm{H}), 8.43(\mathrm{~d}, J$ $=8.1,4 \mathrm{H}), 8.72(\mathrm{~d}, J=8.1,4 \mathrm{H}), 8.82-8.94(\mathrm{~m}, 16 \mathrm{H}) ; \mathrm{MS}_{\left(\mathrm{FAB}^{+}\right)} 2167\left(\mathrm{M}^{+}\right)$; Anal. Calcd for $\mathrm{C}_{148} \mathrm{H}_{118} \mathrm{O}_{10} \mathrm{~N}_{8} \cdot 1 \mathrm{H}_{2} \mathrm{O}: \mathrm{C}, 81.30 ; \mathrm{H}, 5.53 ; \mathrm{N}, 5.12$. Found: $\mathrm{C}, 81.54 ; \mathrm{H}, 5.45 ; \mathrm{N}, 5.19$.

$(S, R, S)-10 b: 83 \%$ yield; $\mathrm{mp}=280-283{ }^{\circ} \mathrm{C} ;[\alpha]_{\mathrm{D}}{ }^{21}=-3290\left(\mathrm{c}=0.05, \mathrm{CHCl}_{3}\right) ; \mathrm{IR}(\mathrm{KBr})$ 2955, 1743, $1602 \mathrm{~cm}^{-1} ;{ }^{1} \mathrm{H}-\mathrm{NMR}\left(300 \mathrm{MHz}, \mathrm{CDCl}_{3}\right) \delta-2.75(\mathrm{~s}, 4 \mathrm{H}), 0.60-0.75(\mathrm{~m}, 12 \mathrm{H})$, 0.9-1.3 (m, 8H), 1.3-1.6 (m, 8H), 3.70-4.25 (m, 8H), $3.77(\mathrm{~s}, 6 \mathrm{H}), 7.20-7.85(\mathrm{~m}, 32 \mathrm{H}), 8.02$ $(\mathrm{d}, J=7.8,2 \mathrm{H}), 8.07(\mathrm{~s}, 2 \mathrm{H}), 8.15-8.28(\mathrm{~m}, 12 \mathrm{H}), 8.42(\mathrm{~d}, J=8.4,4 \mathrm{H}), 8.71(\mathrm{~d}, J=8.4$, 4H), 8.80-9.00 (m, 16H); MS (FAB $\left.{ }^{+}\right) 2167\left(\mathrm{M}^{+}\right)$; Anal. Calcd for $\mathrm{C}_{148} \mathrm{H}_{118} \mathrm{O}_{10} \mathrm{~N}_{8} \cdot 1 \mathrm{H}_{2} \mathrm{O}: \mathrm{C}$, 81.30; H, 5.53; N, 5.12. Found: C, 81.25; H, 5.48; N, 5.14.

$(S, S, S, S, S, S, S)-11 \mathbf{b}: 90 \%$ yield; $\mathrm{mp}=261-263{ }^{\circ} \mathrm{C} ;[\alpha]_{\mathrm{D}}{ }^{21}=-3960\left(\mathrm{c}=0.05, \mathrm{CHCl}_{3}\right) ; \mathrm{IR}$ (KBr) 2955, 1743, $1441 \mathrm{~cm}^{-1}$; ${ }^{1} \mathrm{H}-\mathrm{NMR}\left(300 \mathrm{MHz}, \mathrm{CDCl}_{3}\right) \delta-2.74(\mathrm{~s}, 4 \mathrm{H}), 0.66(\mathrm{t}, J=7.4$, $6 \mathrm{H}), 0.70-0.8(\mathrm{~m}, 18 \mathrm{H}), 0.85-1.6(\mathrm{~m}, 32 \mathrm{H}), 3.80-4.00(\mathrm{~m}, 8 \mathrm{H}), 3.87(\mathrm{~s}, 6 \mathrm{H}), 3.91(\mathrm{~s}, 6 \mathrm{H})$, $3.92(\mathrm{~s}, 6 \mathrm{H}), 4.10-4.35(\mathrm{~m}, 8 \mathrm{H}), 7.20-7.85(\mathrm{~m}, 48 \mathrm{H}), 8.02(\mathrm{~d}, J=8.1,2 \mathrm{H}), 8.09(\mathrm{~s}, 2 \mathrm{H})$, 8.20-8.25 (m, 12H), $8.43(\mathrm{~d}, J=8.4,4 \mathrm{H}), 8.73(\mathrm{~d}, J=8.4,4 \mathrm{H}), 8.84-8.93(\mathrm{~m}, 16 \mathrm{H})$; MS $\left(\mathrm{FAB}^{+}\right) 3079\left(\mathrm{M}^{+}\right)$; Anal. Calcd for $\mathrm{C}_{208} \mathrm{H}_{182} \mathrm{O}_{18} \mathrm{~N}_{8} \cdot 2 \mathrm{H}_{2} \mathrm{O}$ : C, 80.13; H, 6.01; N, 3.59 . Found: C, 80.29; H, 6.02; N, 3.52 . 
${ }^{1} \mathrm{H}-\mathrm{NMR}$ charts for compounds $\mathbf{1 a - 7 a}$ and $\mathbf{9 a - 1 1 a . ~}$ 


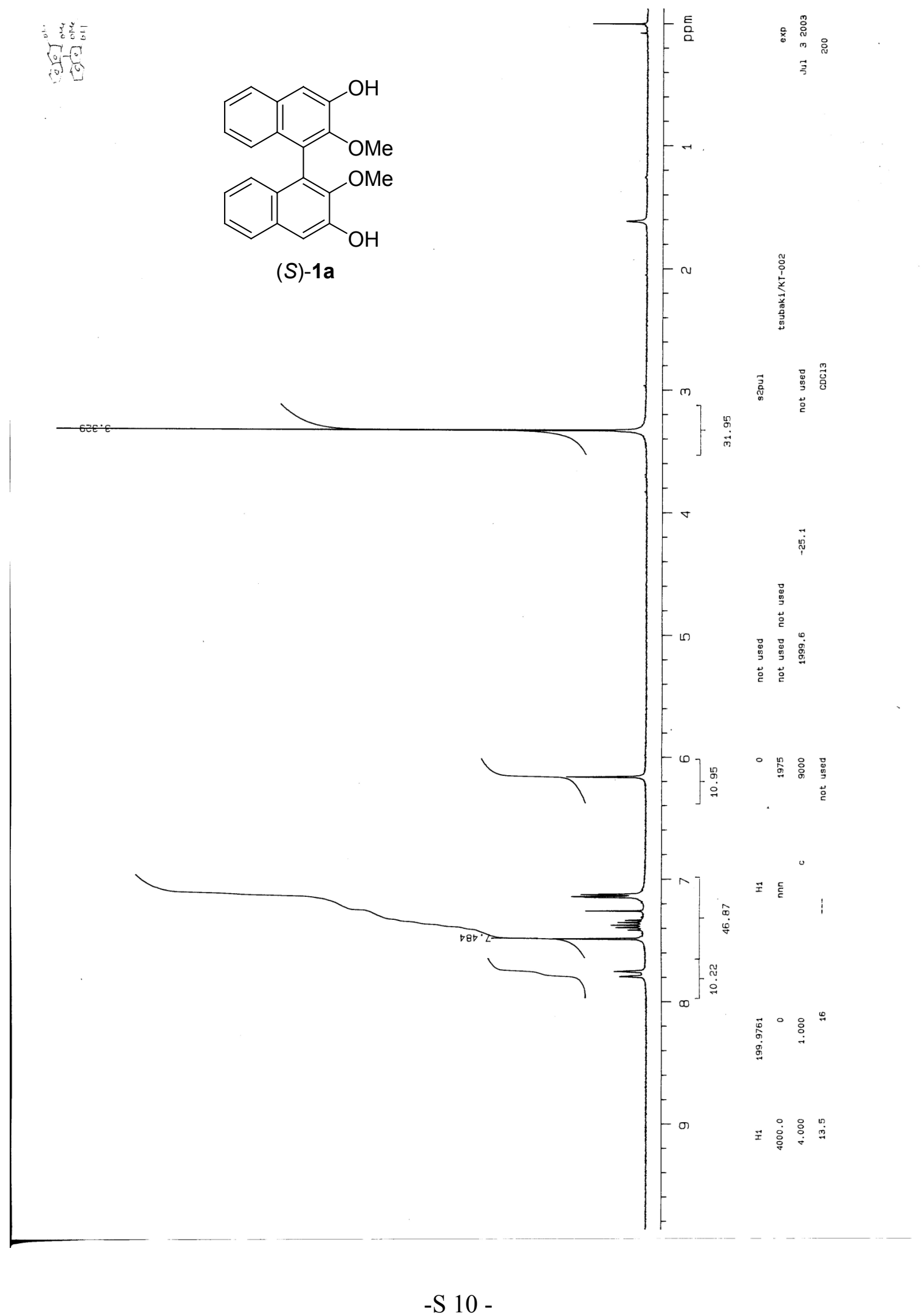




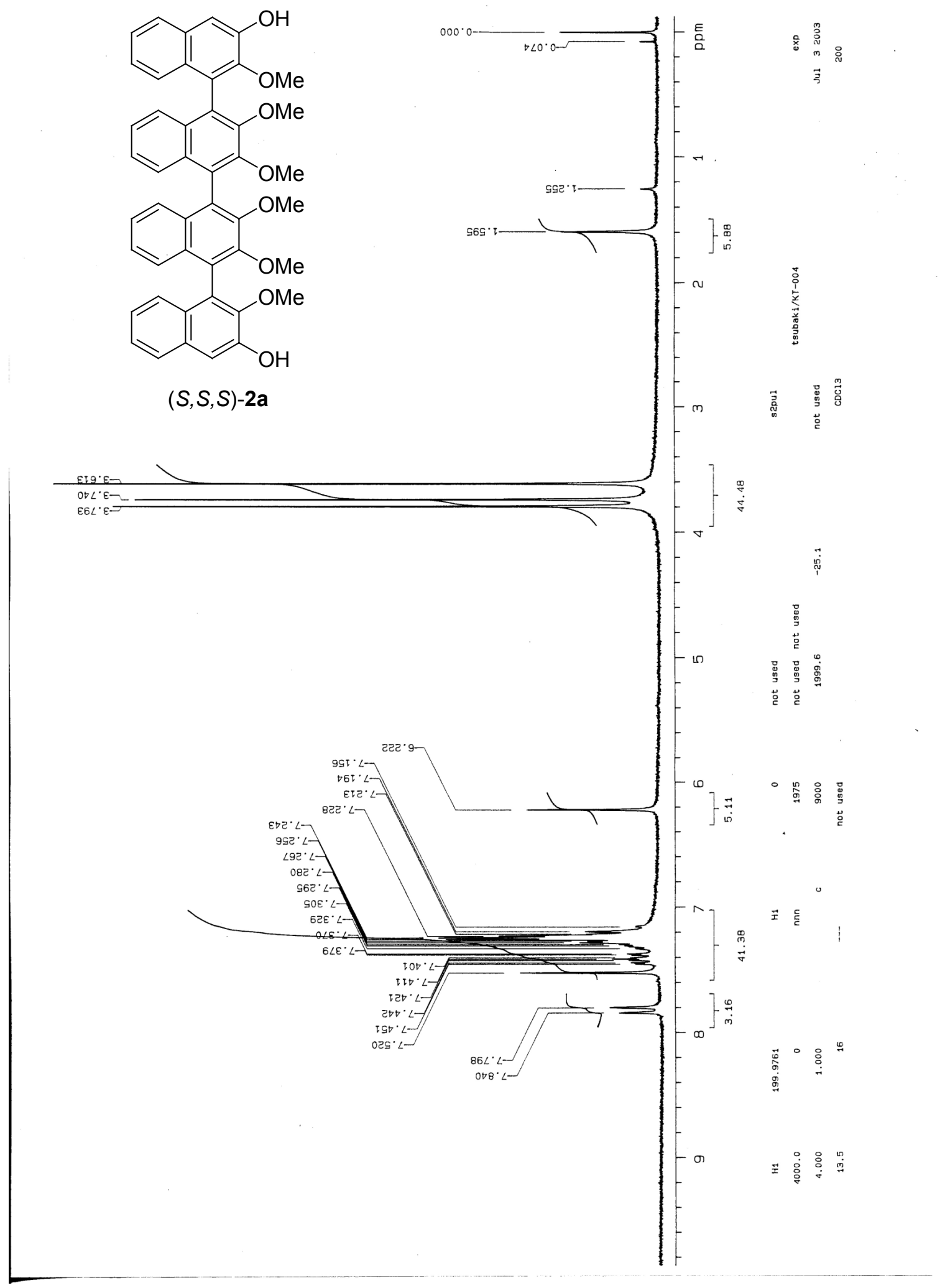

-S 11 - 

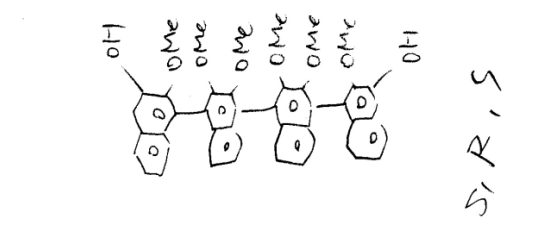<smiles>COc1c(O)cc2ccccc2c1-c1c(OC)c(OC)c2ccccc2c1-c1c(OC)c(OC)c(-c2c(OC)c(O)cc3ccccc23)c2ccccc12</smiles>

$(S, R, S)-\mathbf{2 a}$

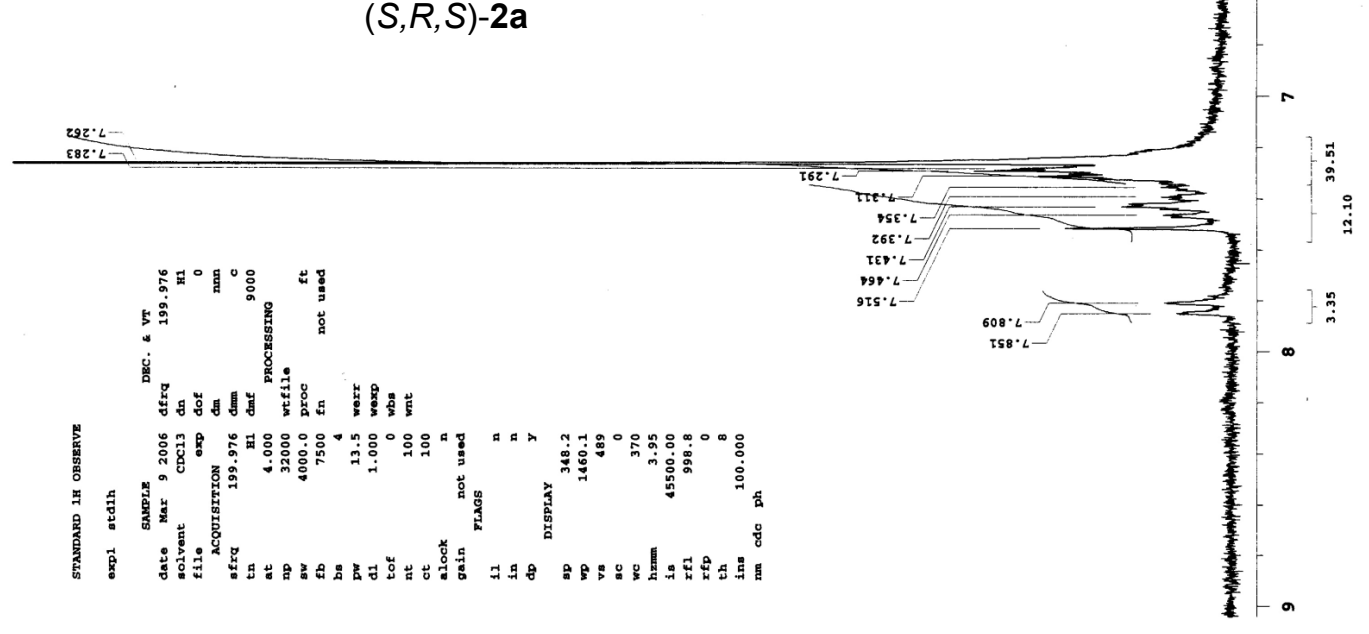




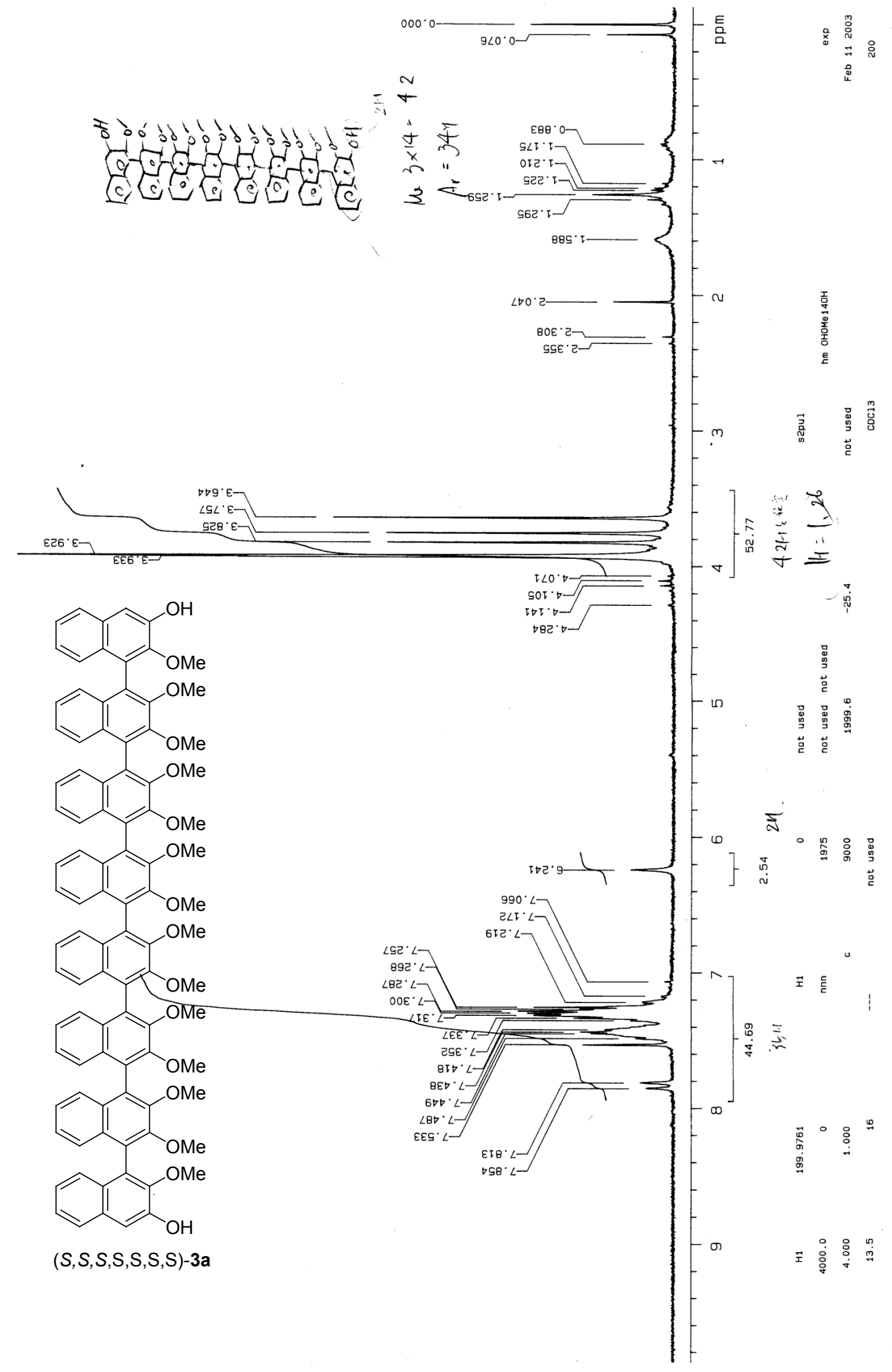




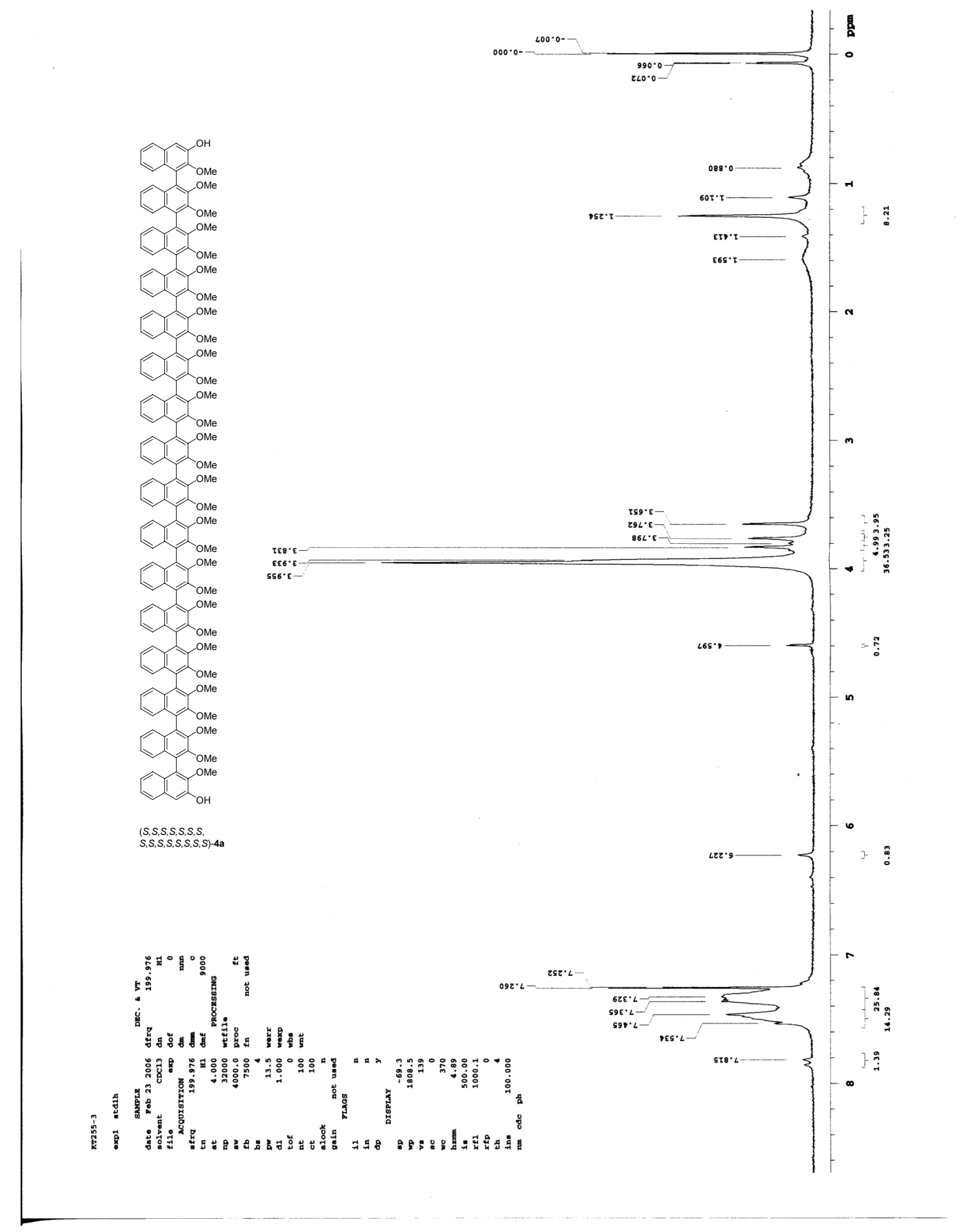




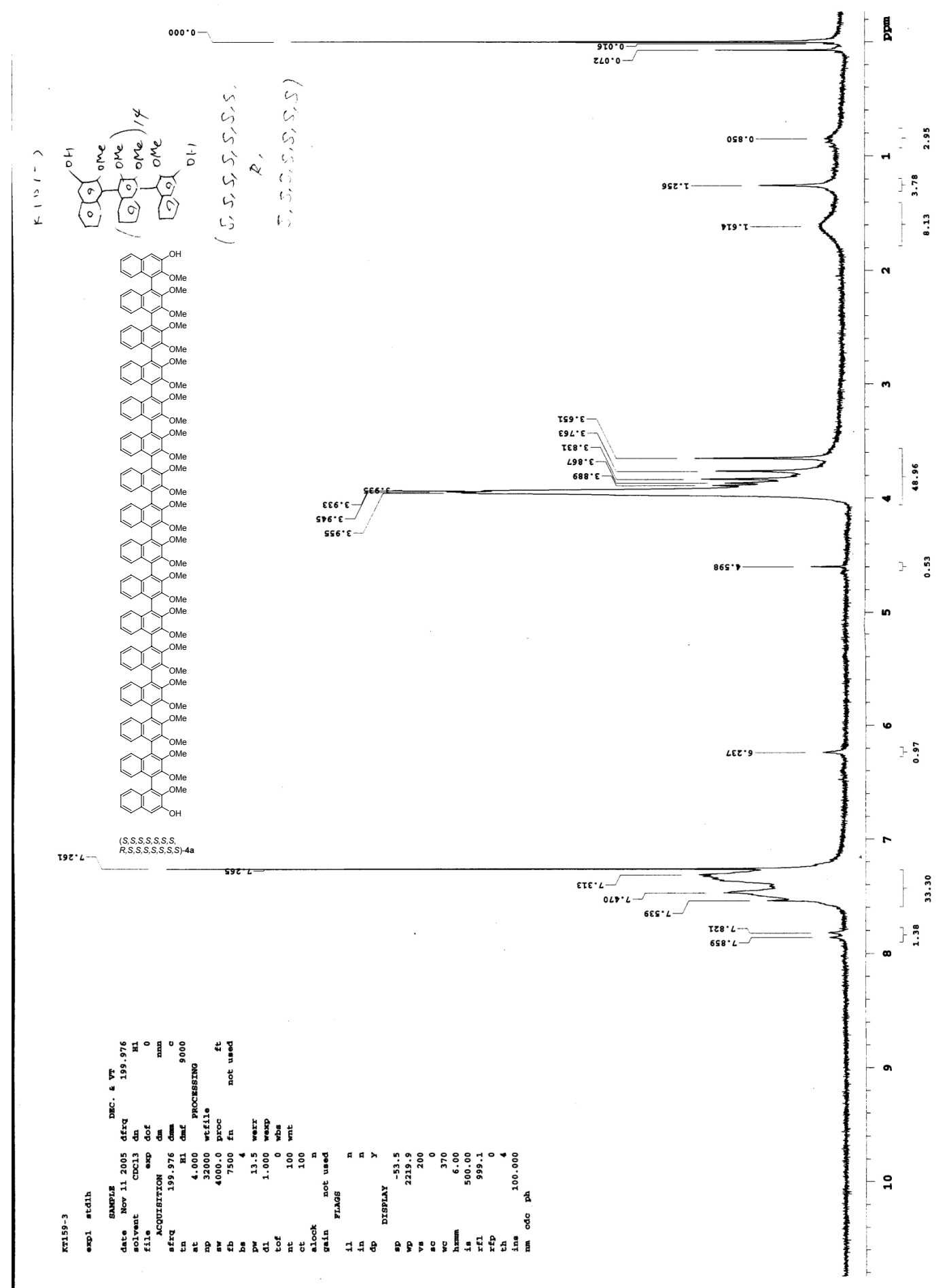




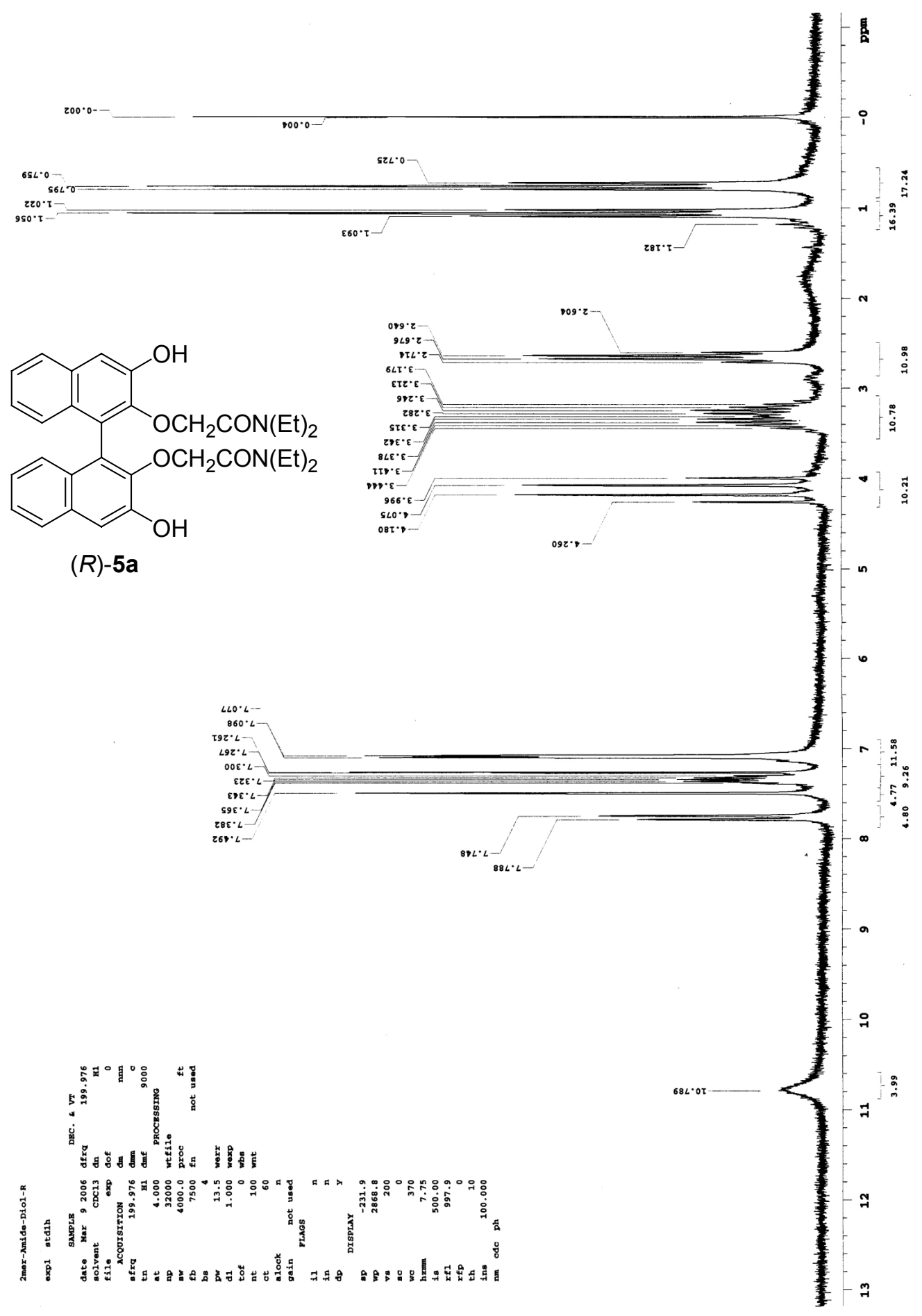




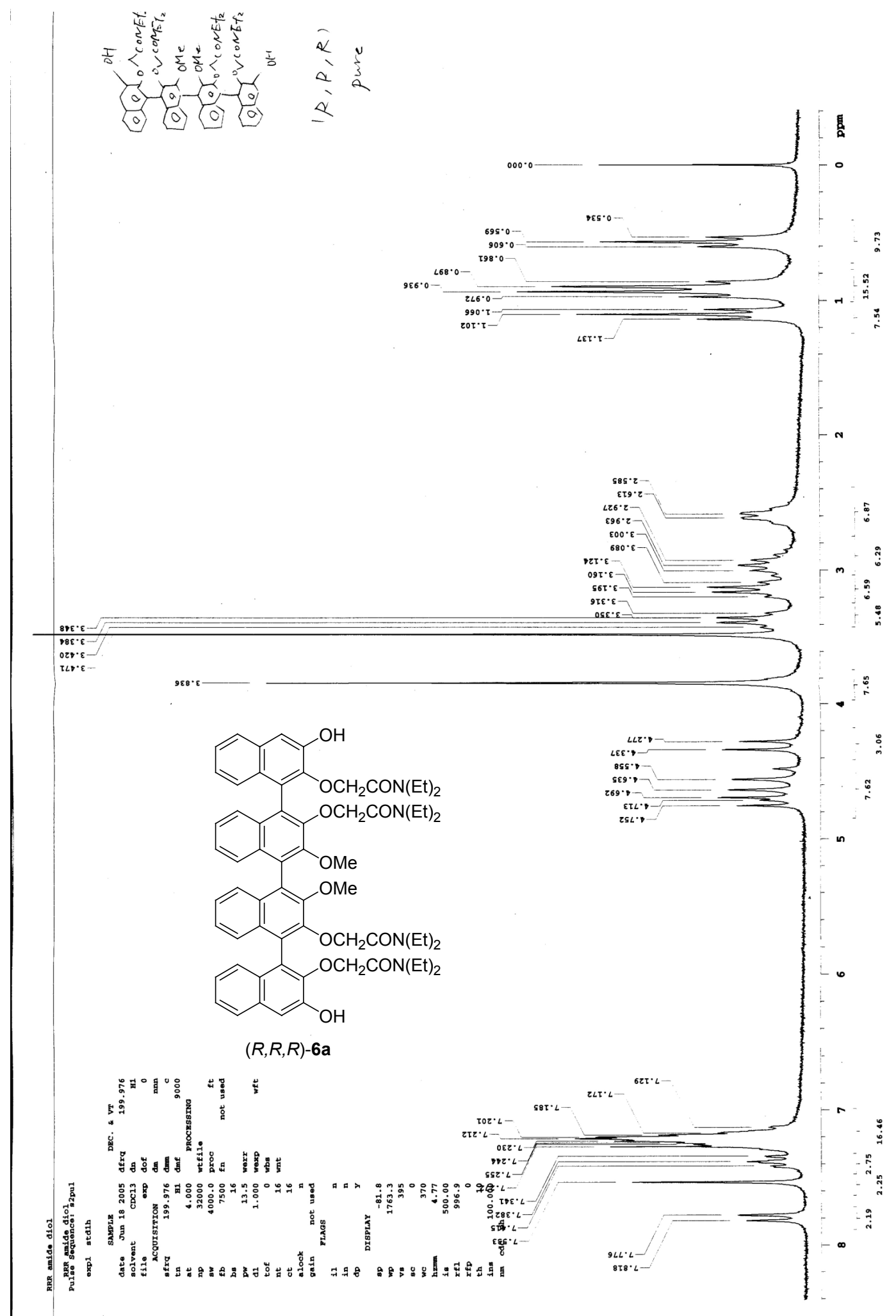




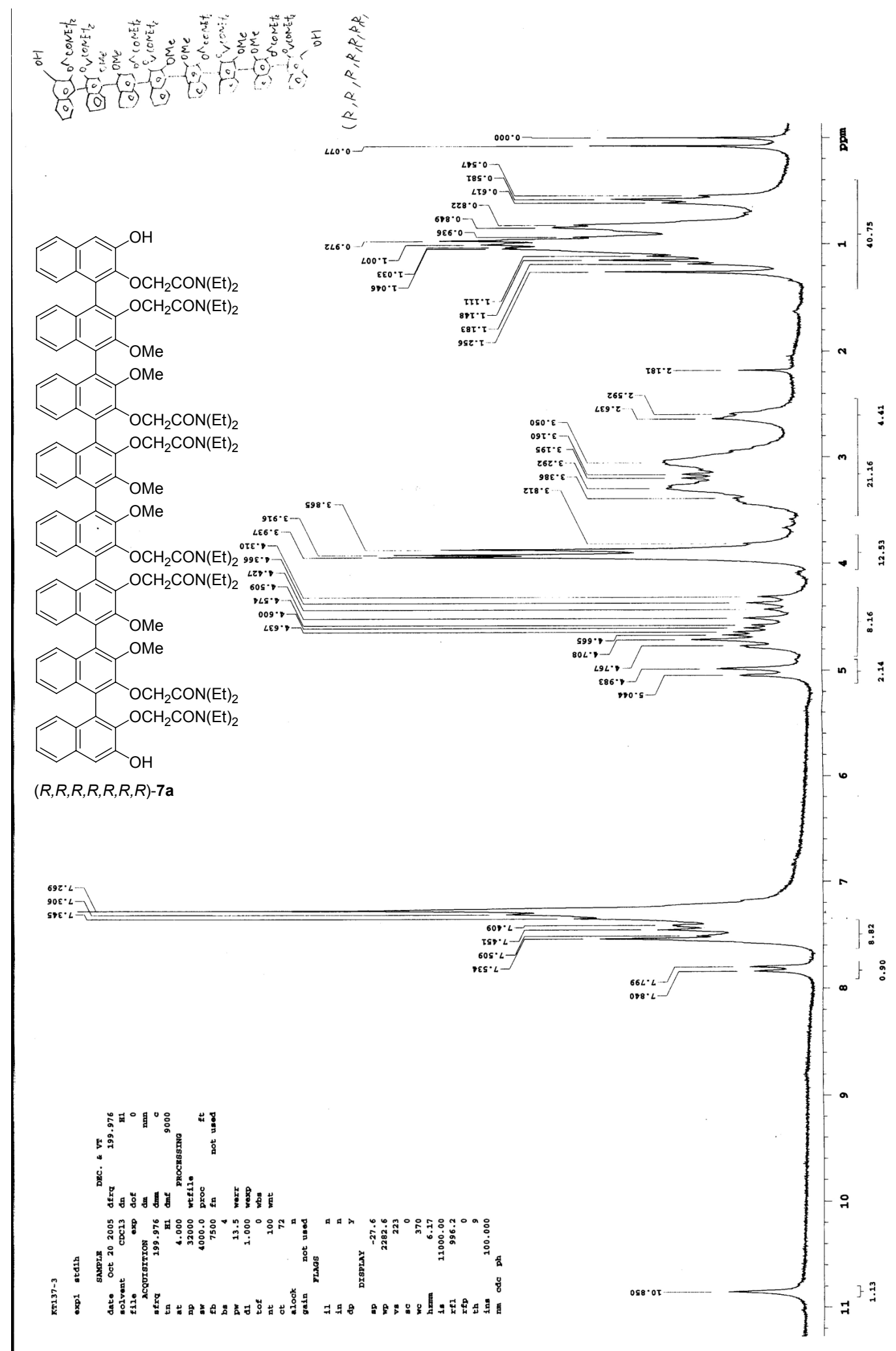




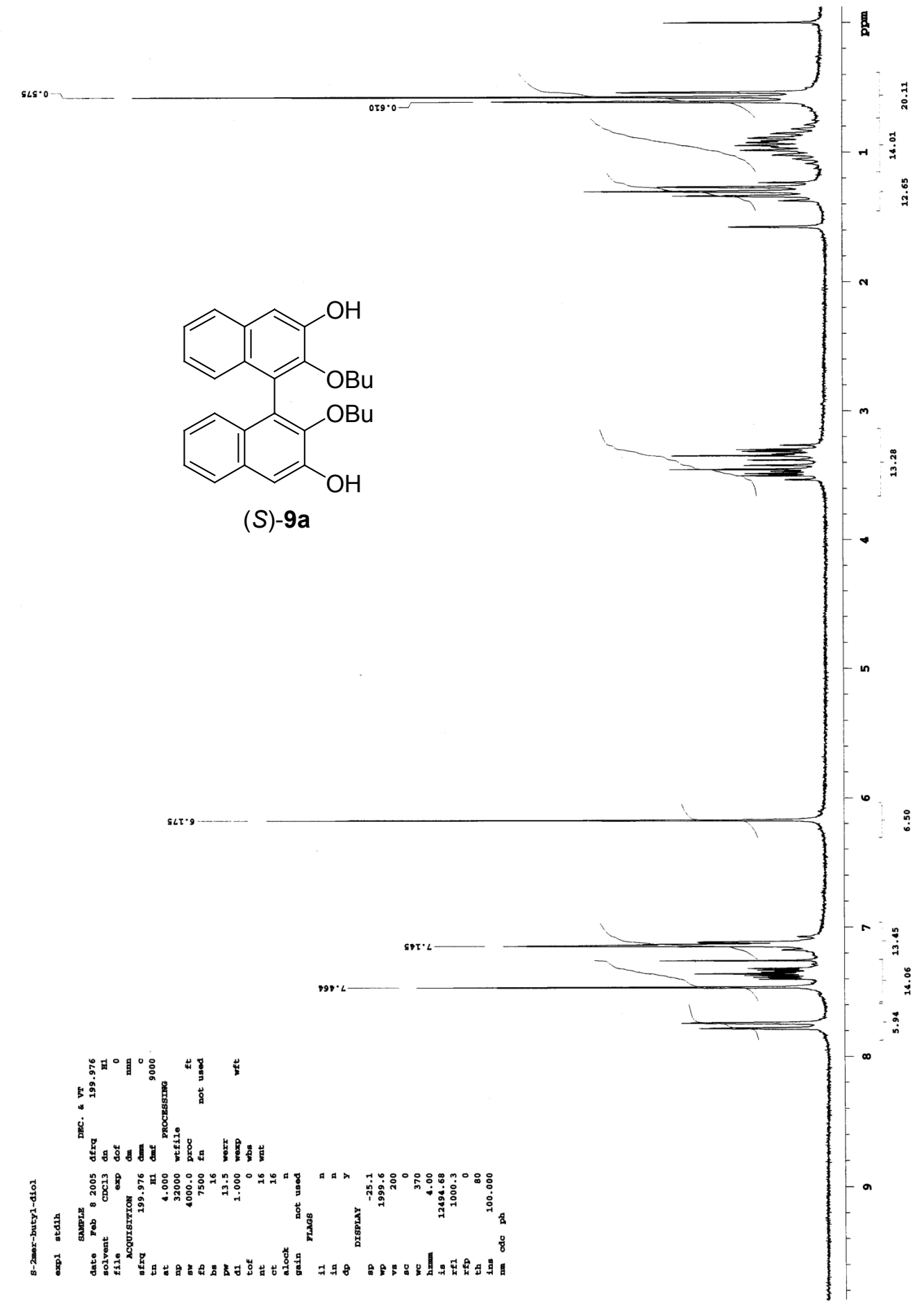



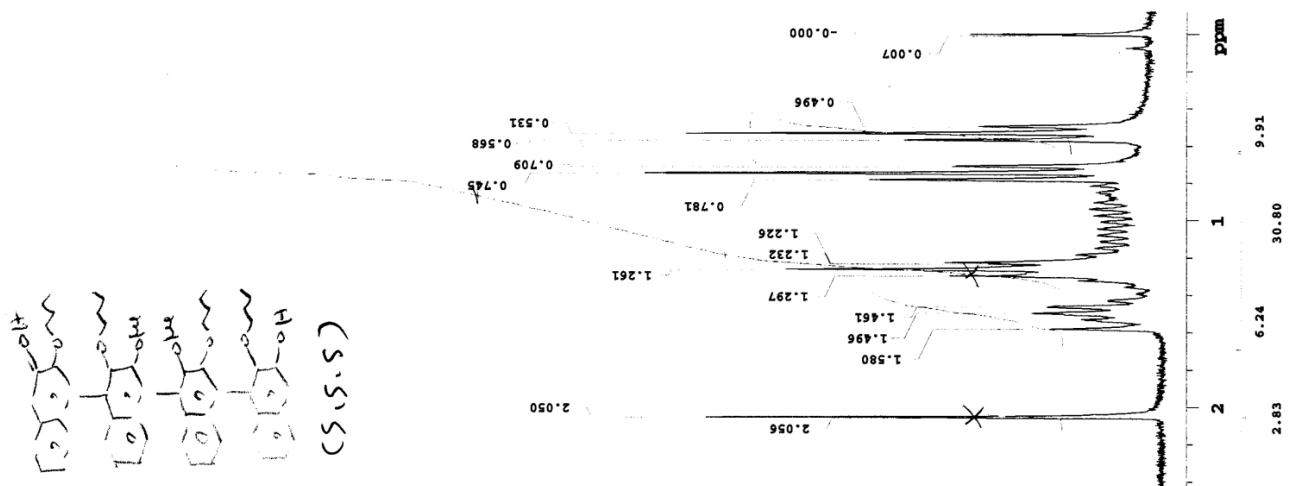

$494 \cdot 8$<smiles>CCCCOc1c(OCC)c(-c2c(OCC)c(O)cc3ccccc23)c(-c2c(OCC)c(O)cc3ccccc23)c2ccccc12</smiles>

(S,S,S)-10a

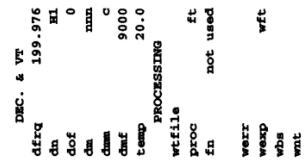

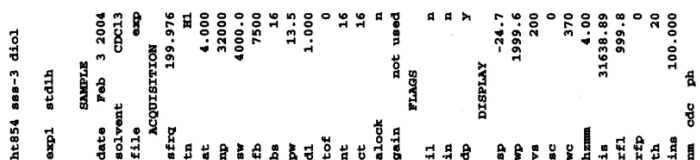

เซโ $ค 9$

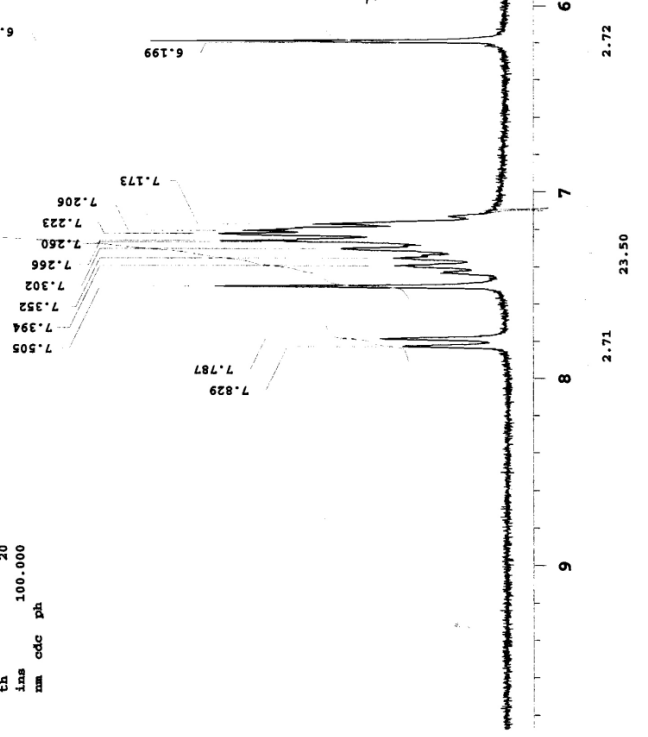




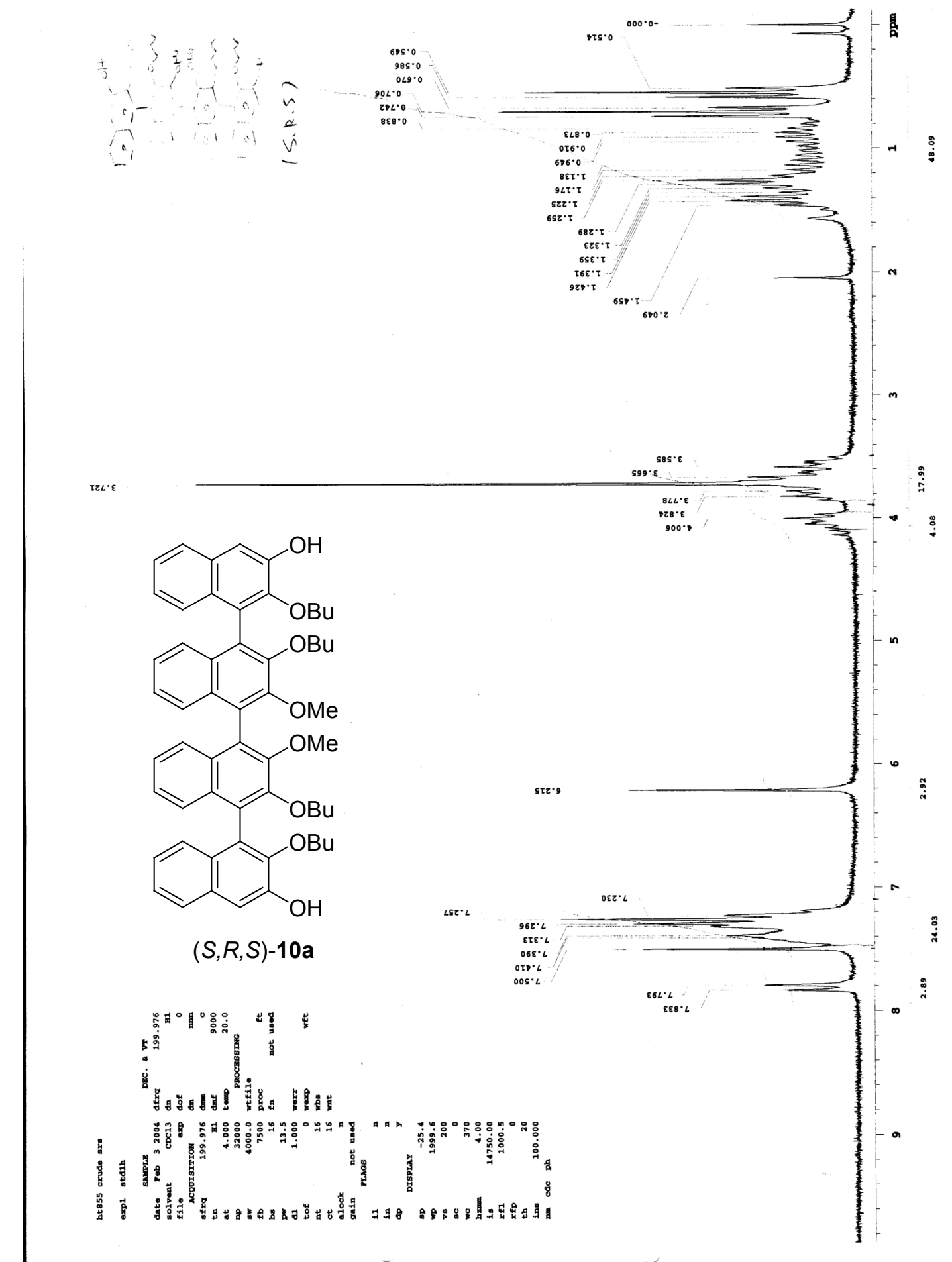




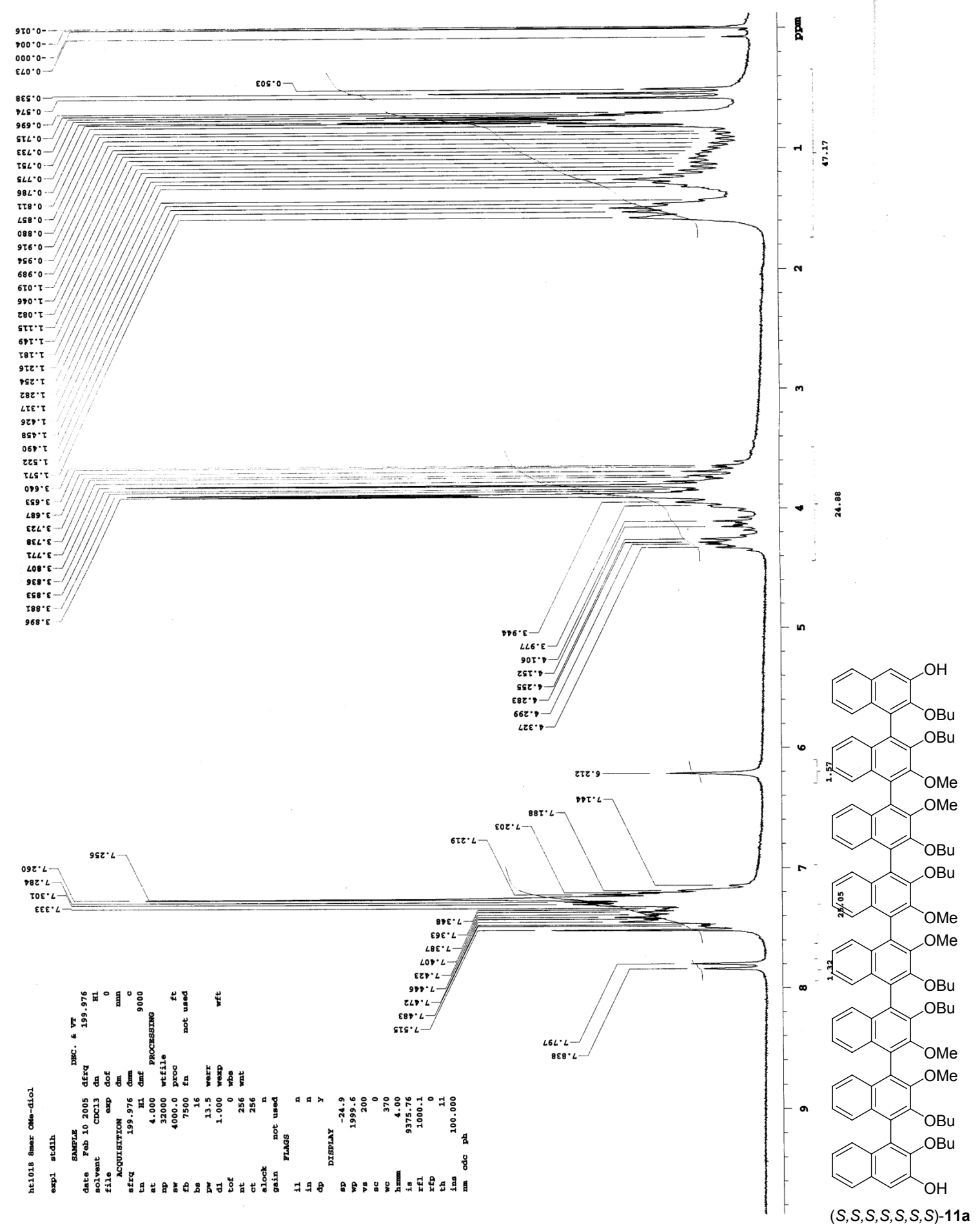


${ }^{1} \mathrm{H}-\mathrm{NMR}$ charts for compounds $\mathbf{1 b}-\mathbf{7} \mathbf{b}$ and $\mathbf{9 b}-\mathbf{1 1 b}$. 


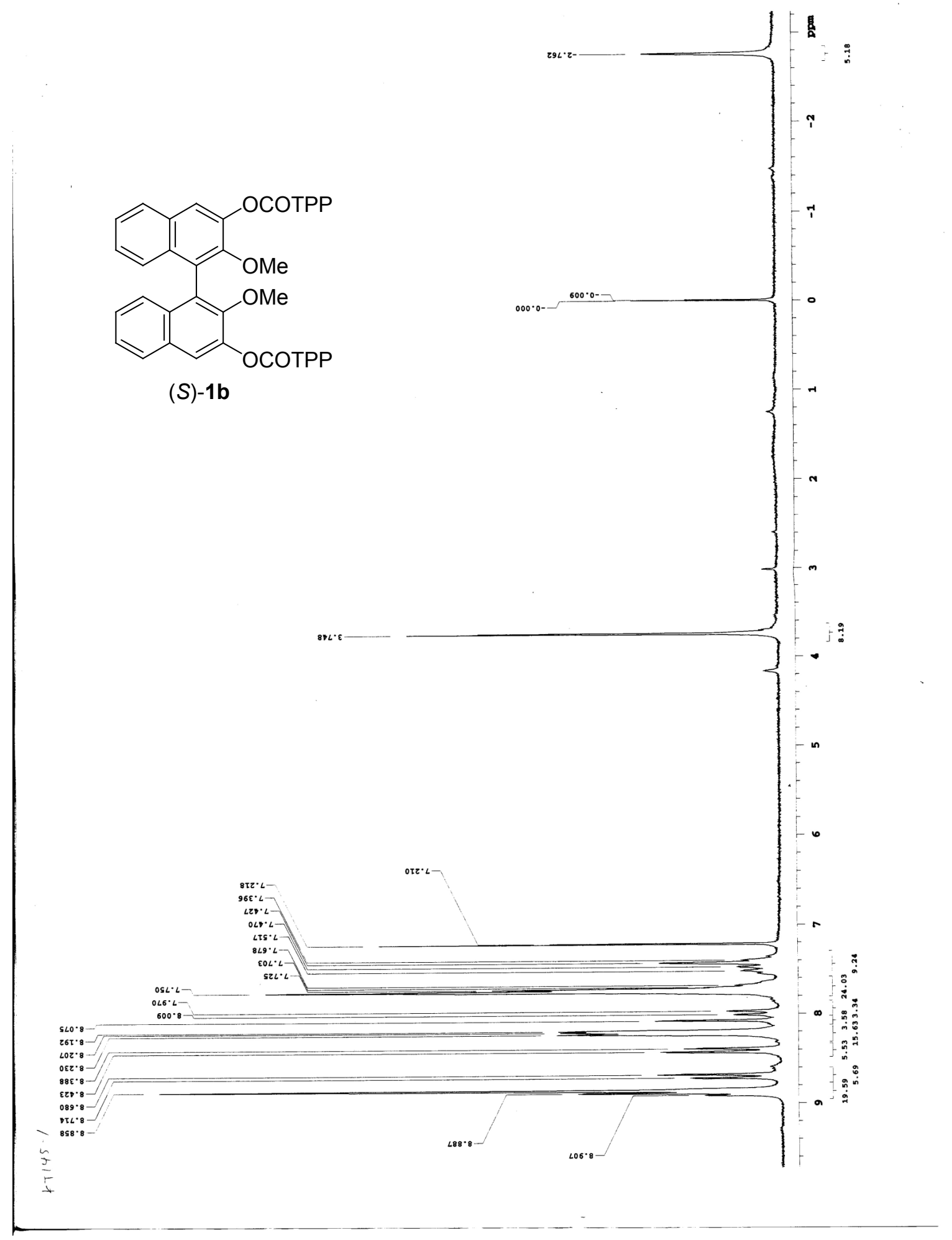

-S 24 - 


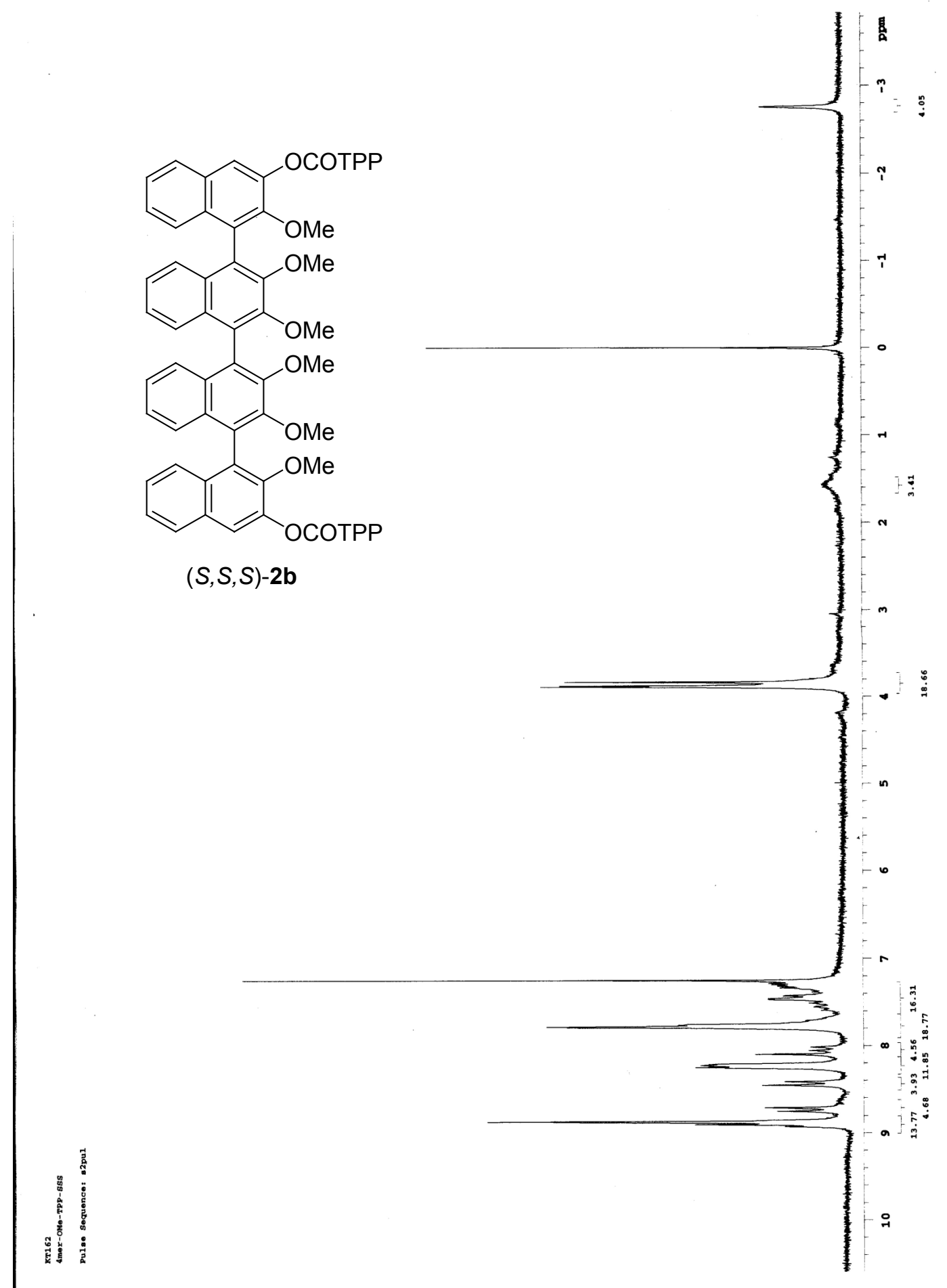



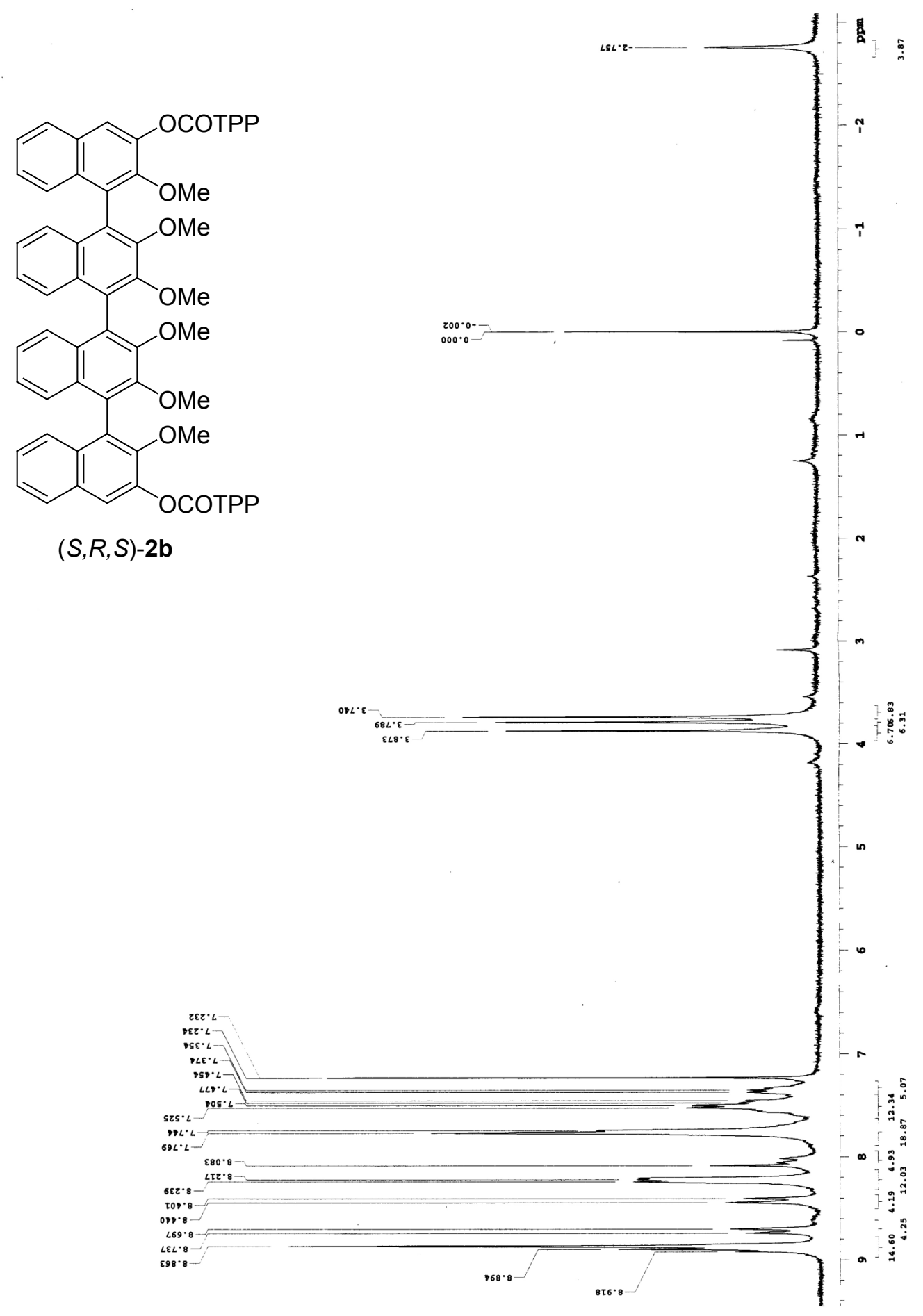

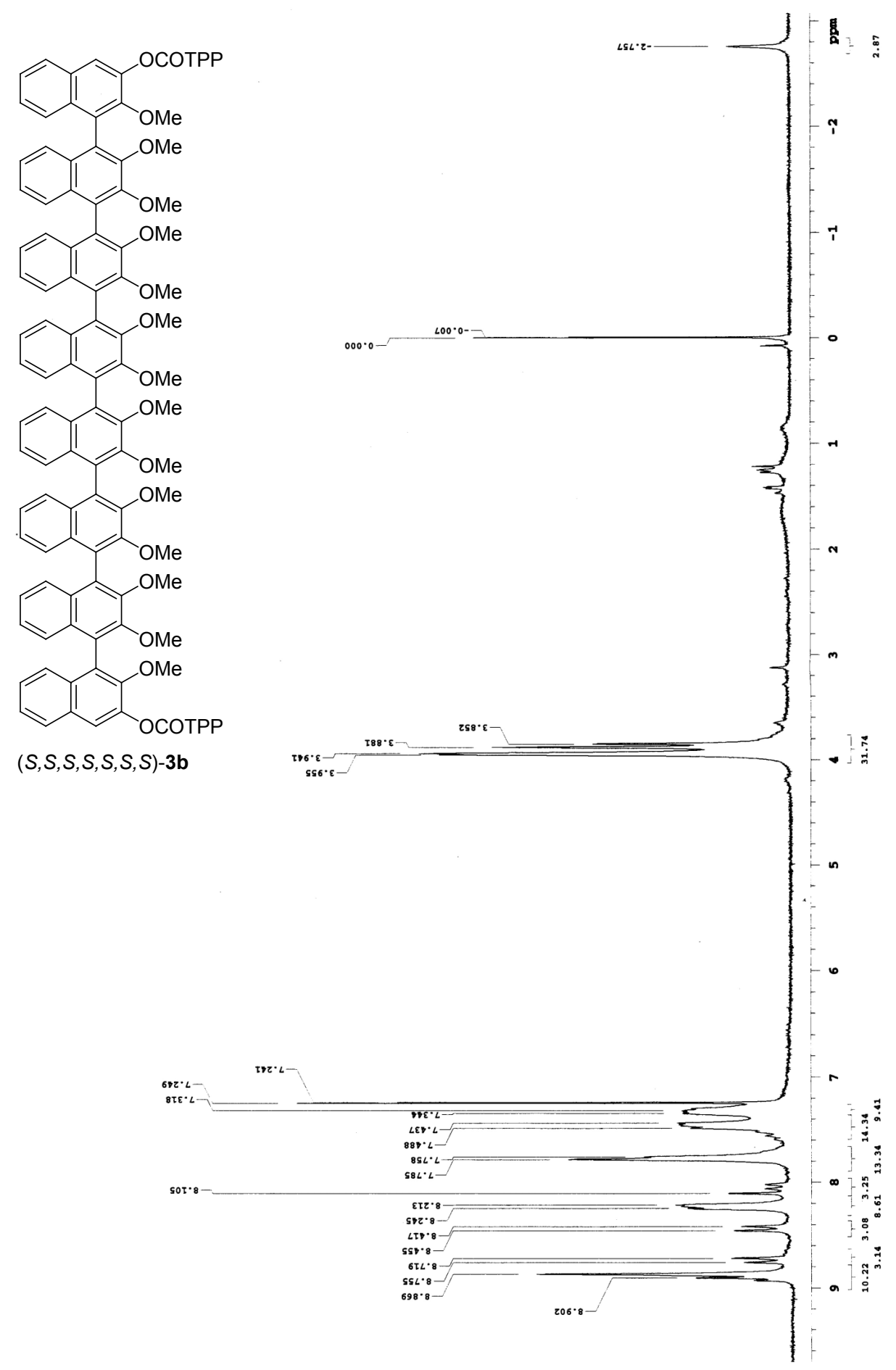


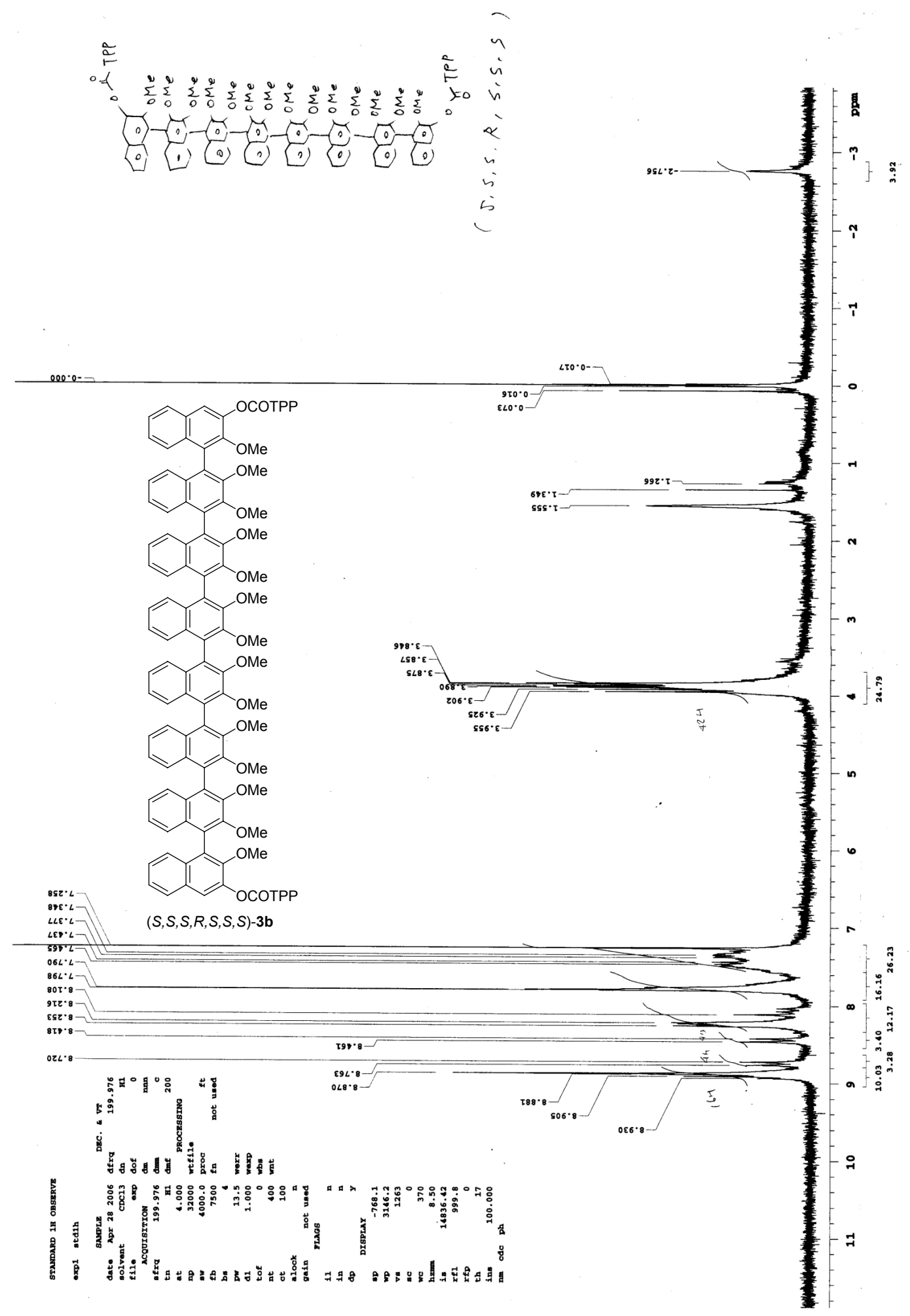




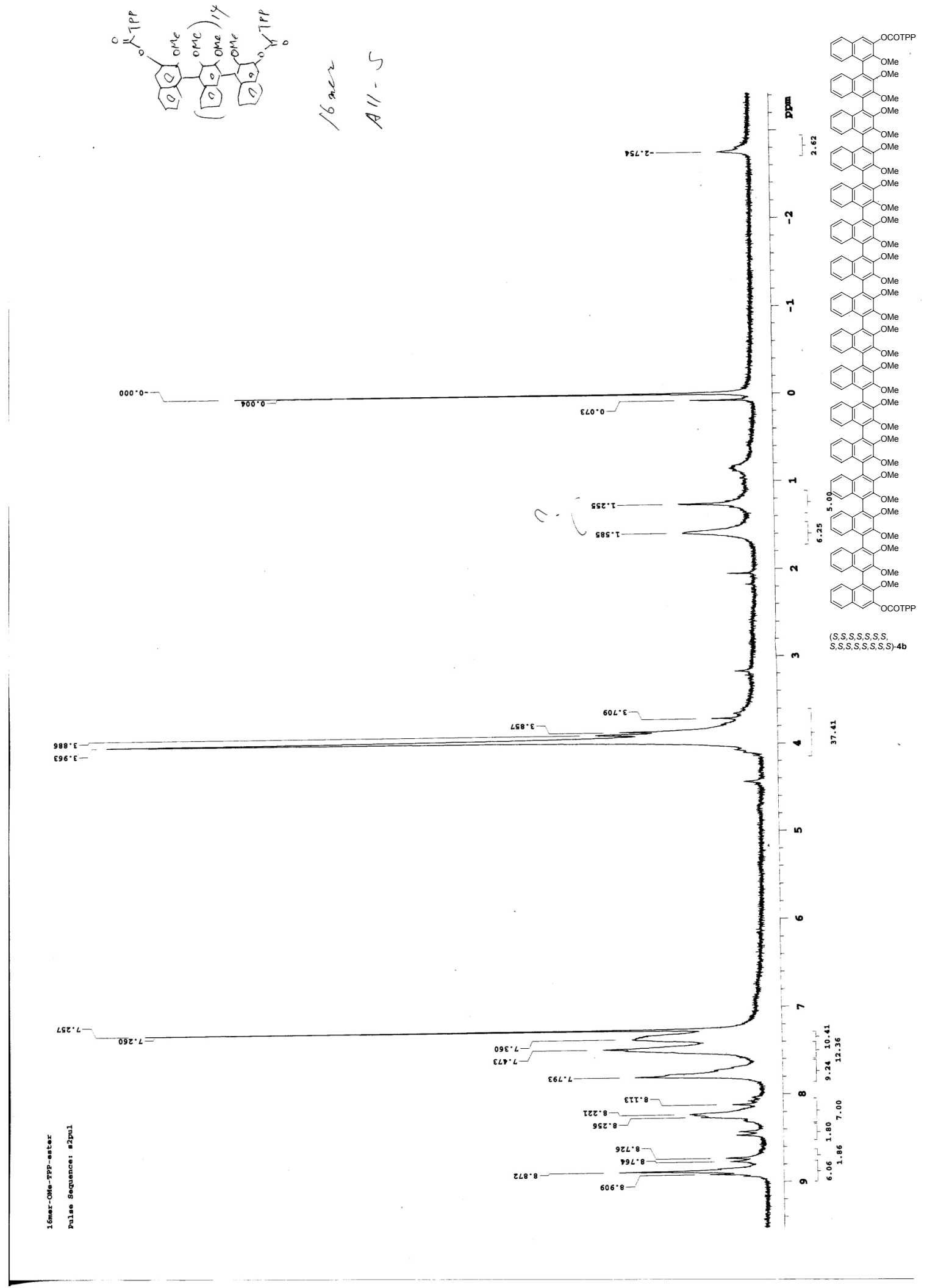



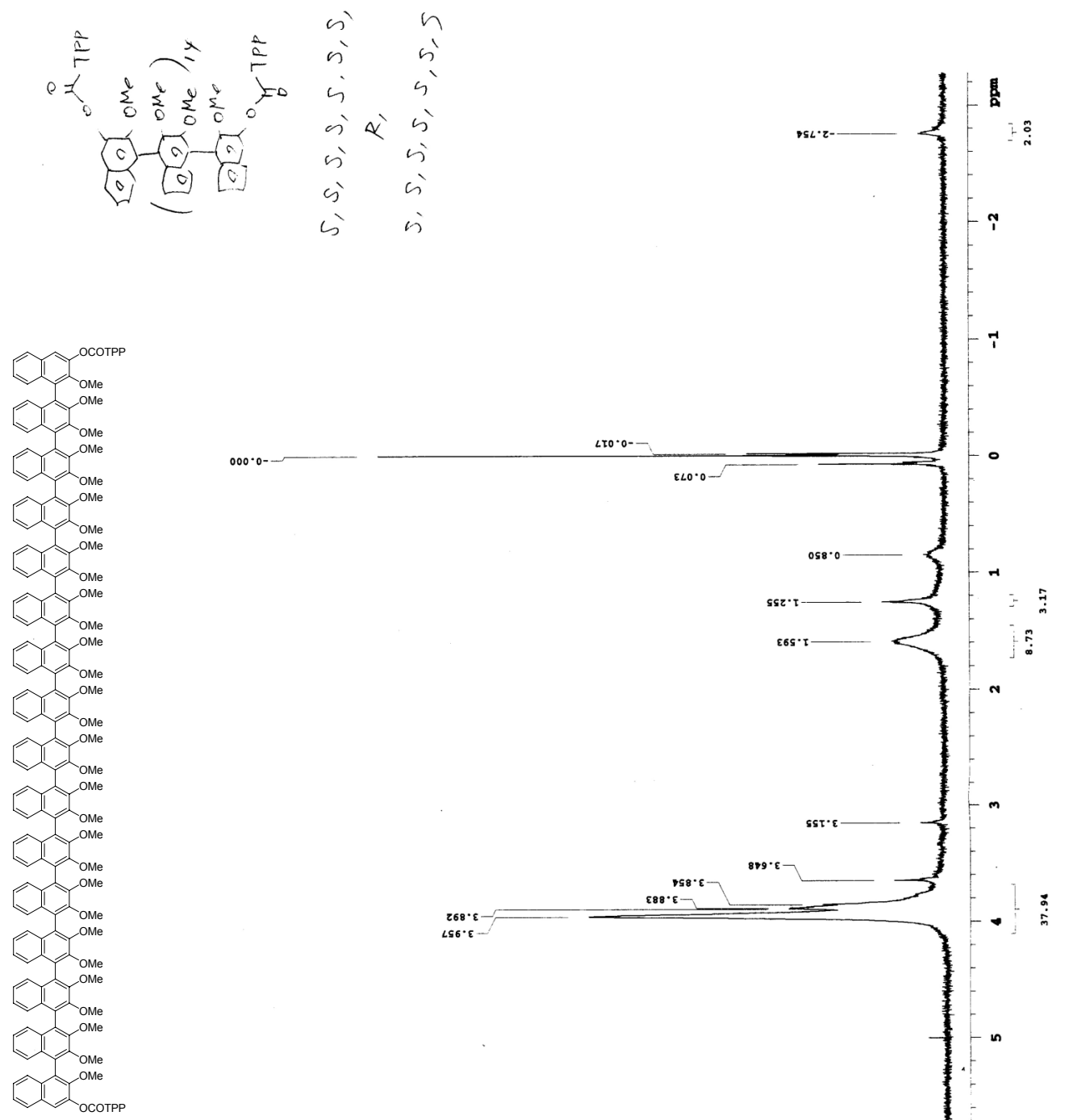

$(S, S, S, S, S, S, S$,
$R, S, S, S, S, S, S, S)-4 b$ 


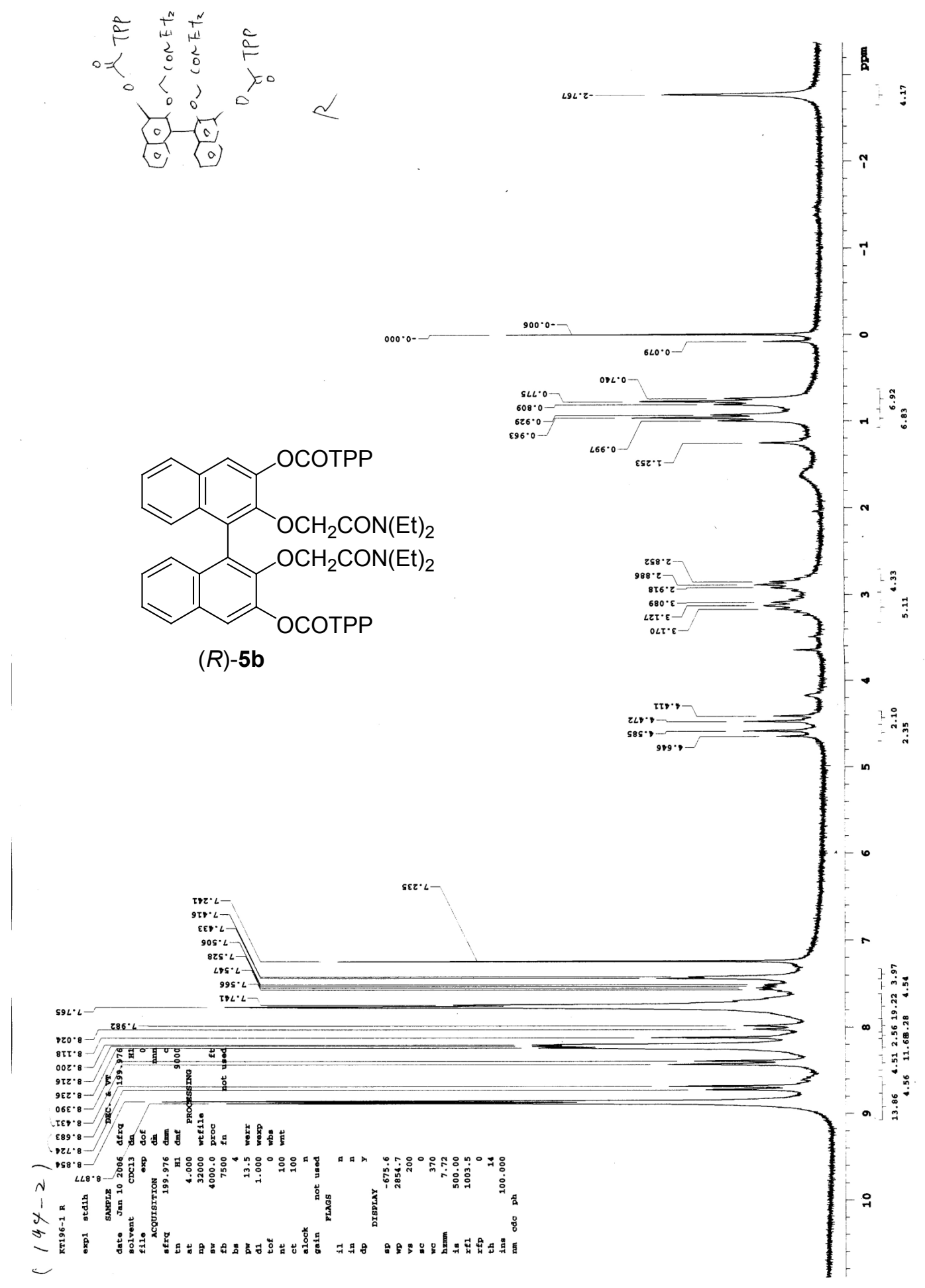




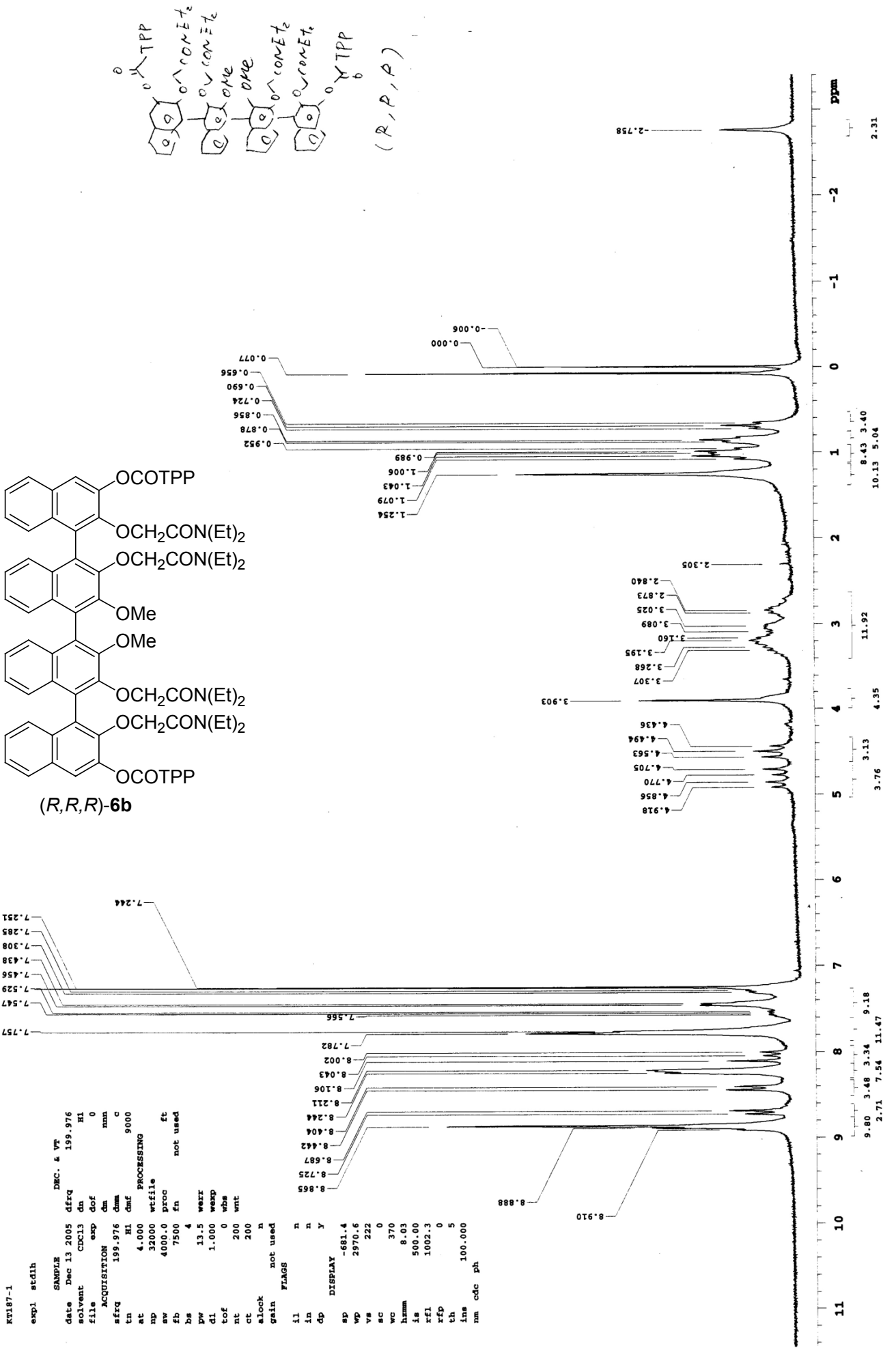




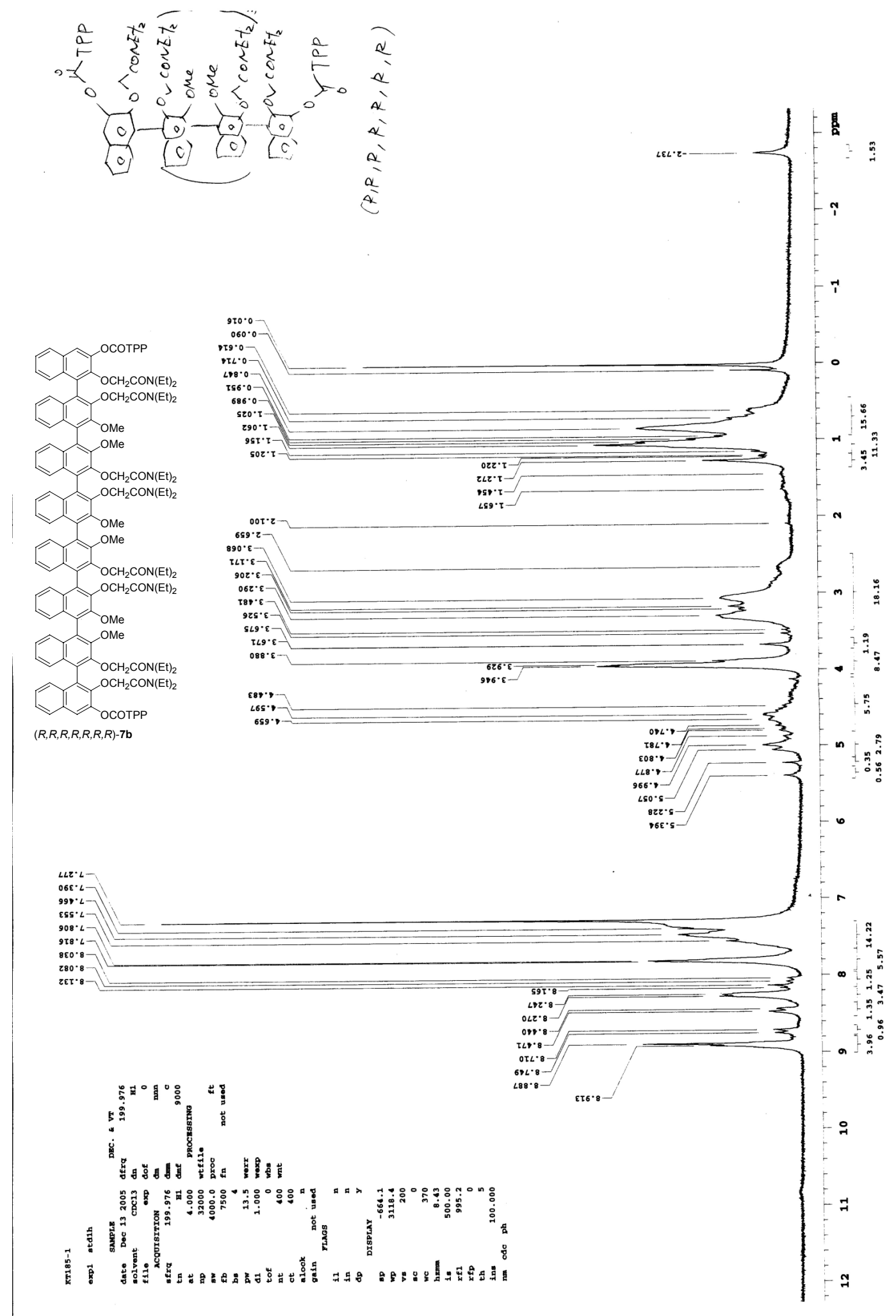




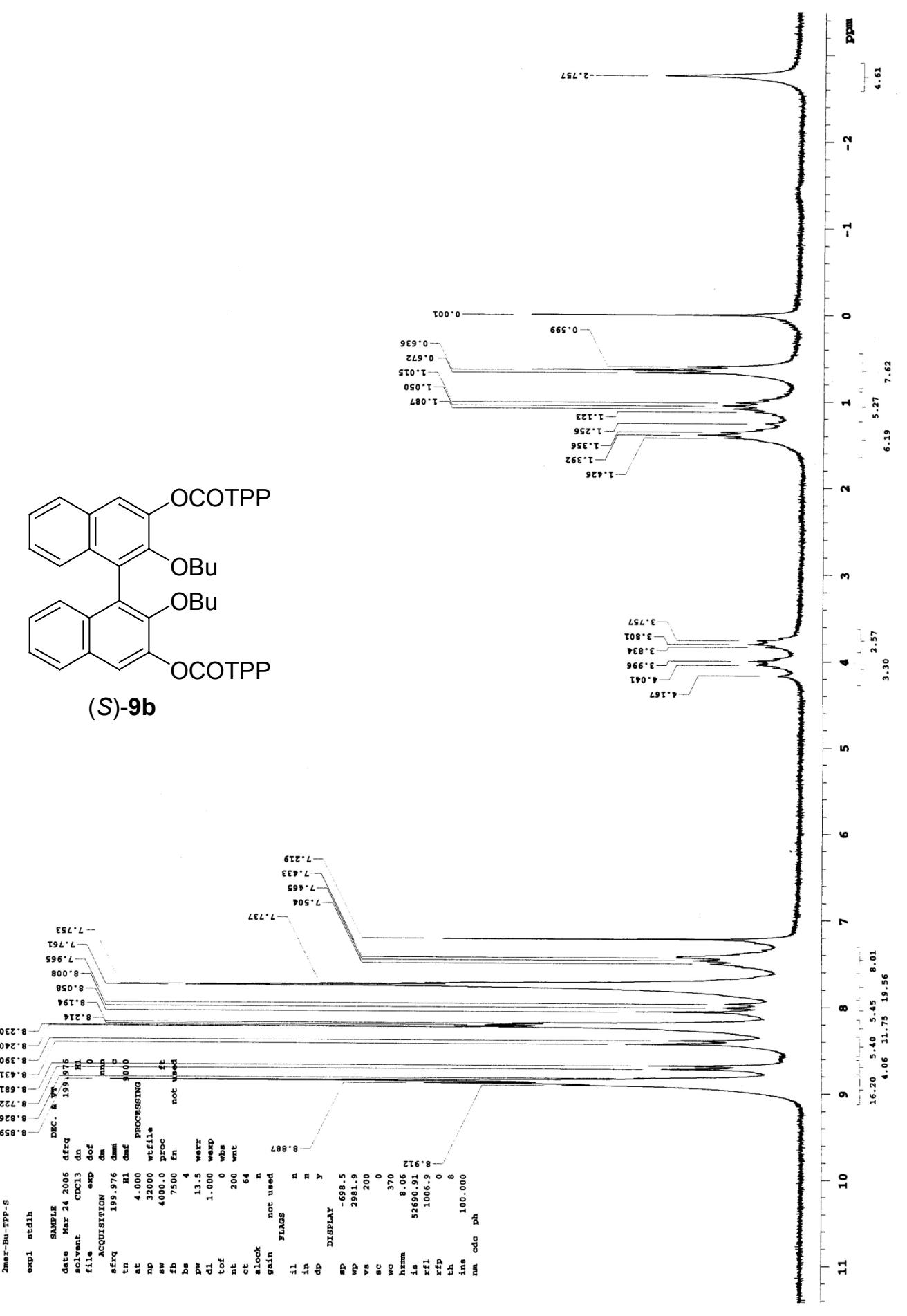



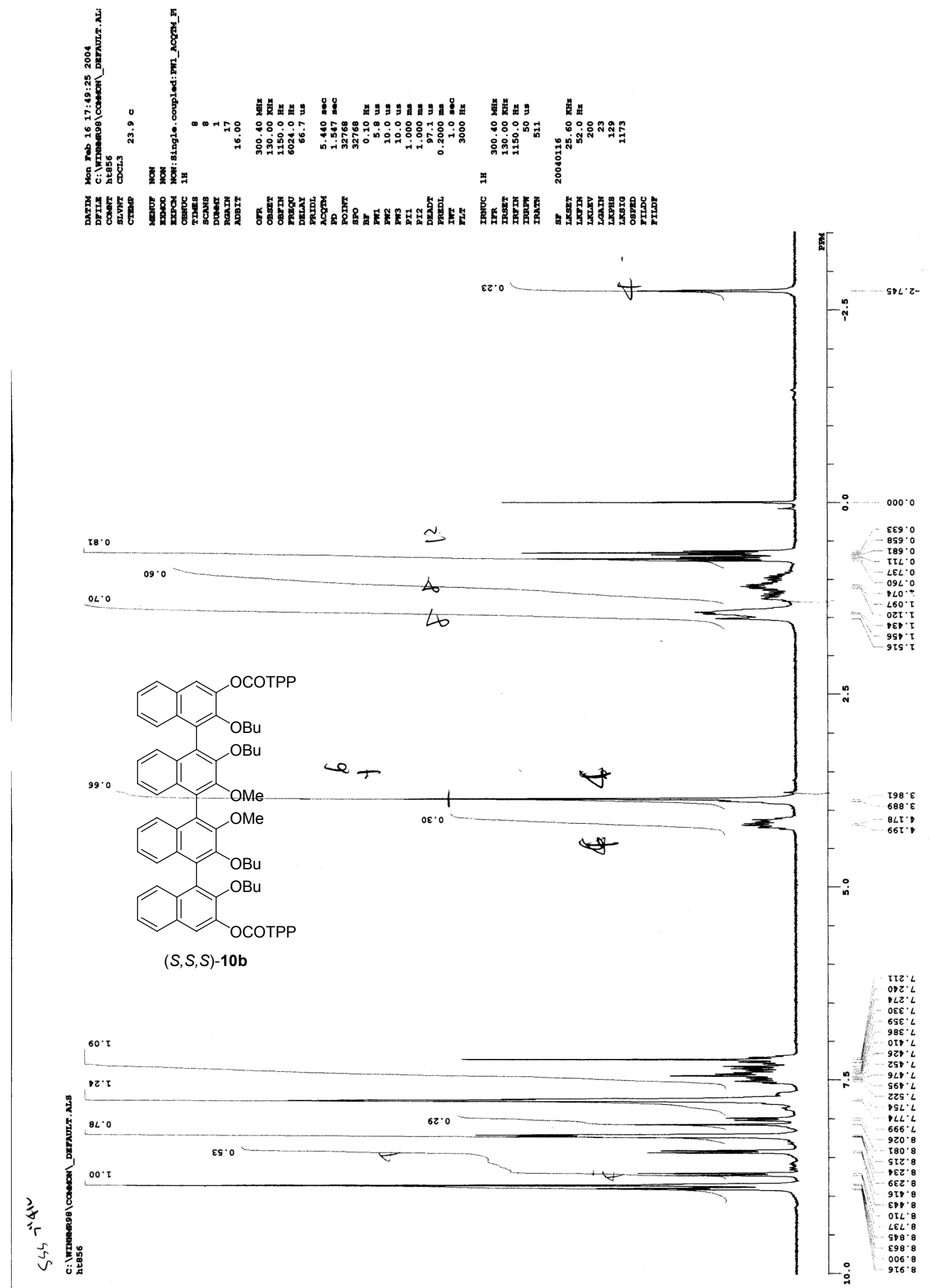

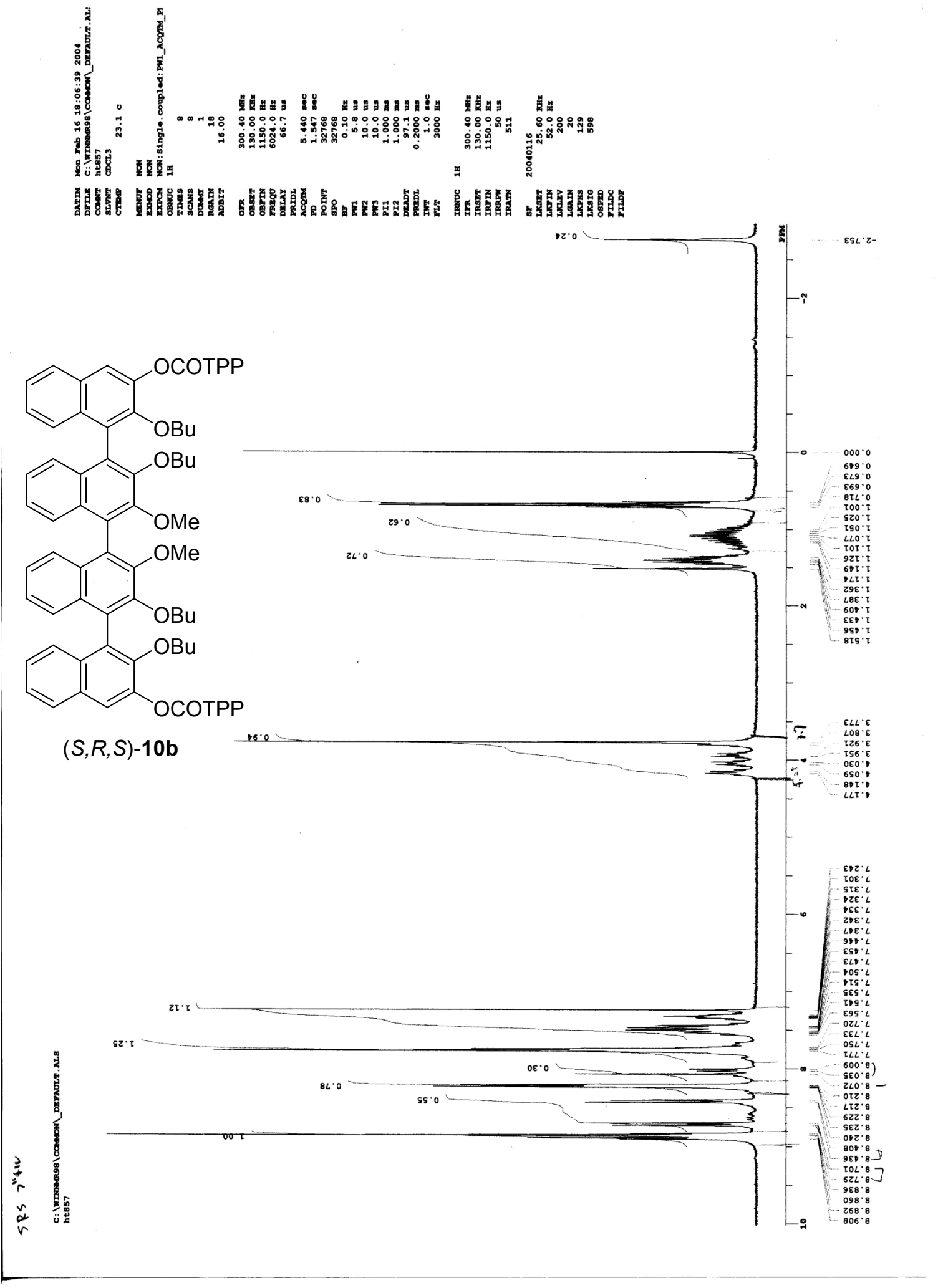


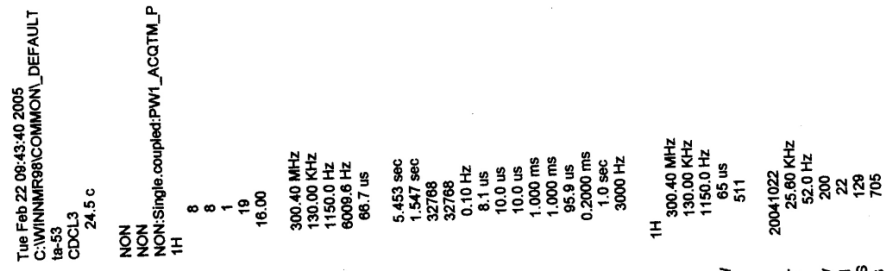

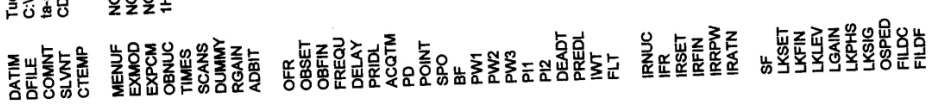

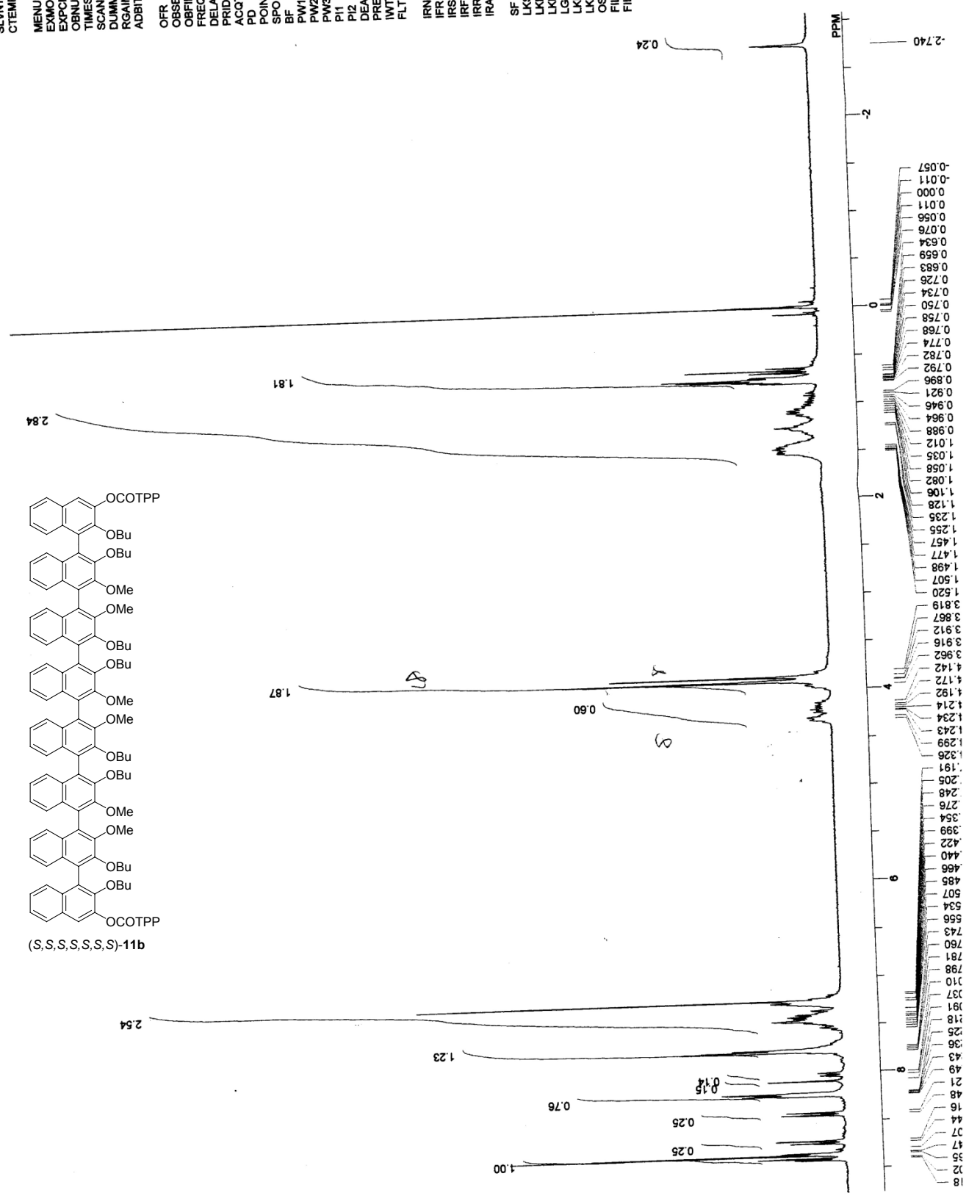

謁 


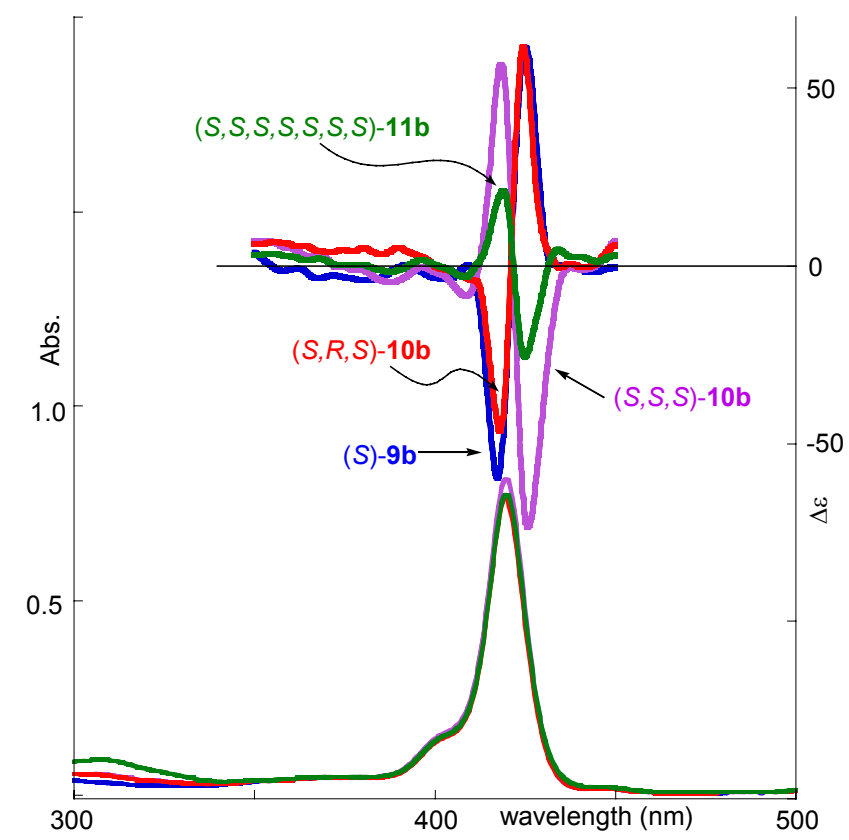

Figure S-1. UV-vis and CD spectra of $\mathbf{9 b}-\mathbf{1 1 b}$ : conditions: $\mathrm{CHCl}_{3}, 1.0 \times 10^{-5} \mathrm{M}, 25^{\circ} \mathrm{C}$, light path length $=1 \mathrm{~mm}$.

\begin{tabular}{lcccccc}
\multirow{2}{*}{ Compound } & \multicolumn{1}{c}{$[\alpha]_{\mathrm{D}}$} & \multicolumn{2}{c}{$\mathrm{UV}$} & \multicolumn{3}{c}{$\mathrm{CD}$} \\
\cline { 2 - 7 } & $\left(\mathrm{CHCl}_{3}\right)$ & $\lambda_{\max }$ & $\varepsilon$ & $\lambda$ & $\Delta \varepsilon$ & $\mathrm{A}_{\mathrm{CD}}{ }^{\mathrm{a}}$ \\
\hline$(S)-\mathbf{9 b}$ & -3850 & 419.5 & 771000 & 425 & 61.5 & 121.2 \\
& & & & 417 & -59.7 & \\
$(S, S, S)-\mathbf{1 0 b}$ & -3360 & 419.5 & 813000 & 426 & -73.4 & 130.1 \\
& & & & 418 & 56.7 & \\
$(S, R, S)-\mathbf{1 0 b}$ & -3290 & 419.5 & 767000 & 425 & 61.6 & 108.1 \\
& & & & 418 & -46.5 & \\
$(S, S, S, S, S, S, S)-\mathbf{1 1 b}$ & -3950 & 419.5 & 772000 & 425 & -25.6 & 46.7 \\
& & & & 419 & 21.1 & \\
& & & & 418 & -21.7 &
\end{tabular}

Table S-2. UV-vis and CD data of 9b-11b. conditions: $\mathrm{CHCl}_{3}, 1.0 \times 10^{-5} \mathrm{M}, 25^{\circ} \mathrm{C}$, light path length $=1 \mathrm{~mm}$. (a) total amplitude of the CD. 
Figure S-2. X-Ray Structures of Ternaphthalenes.
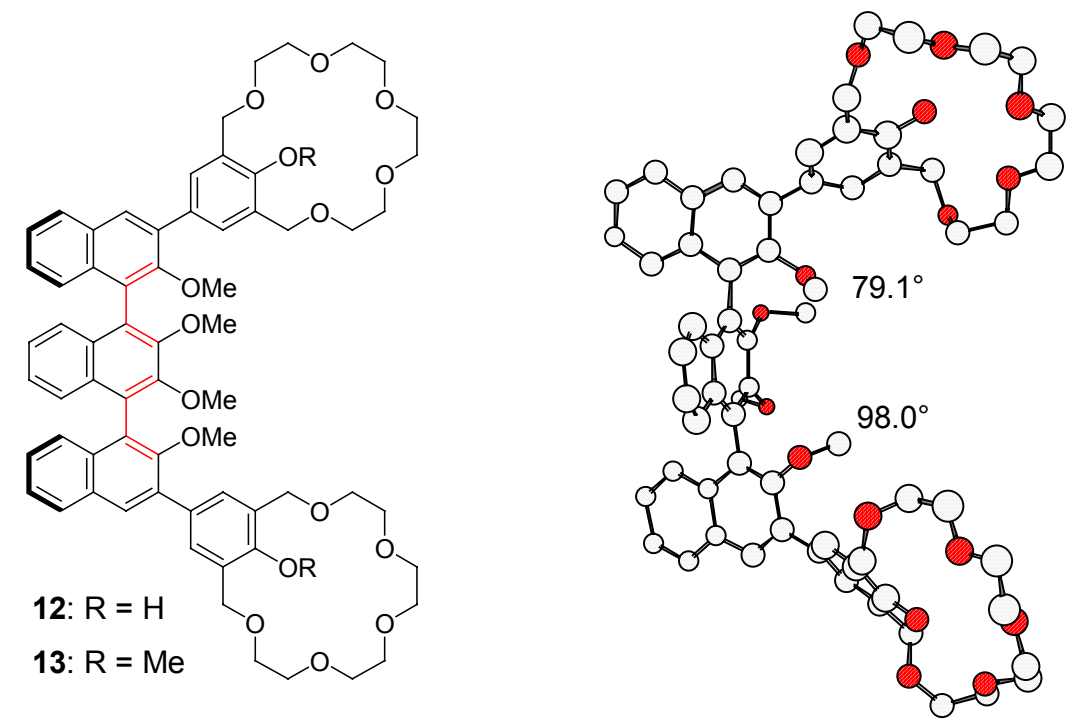

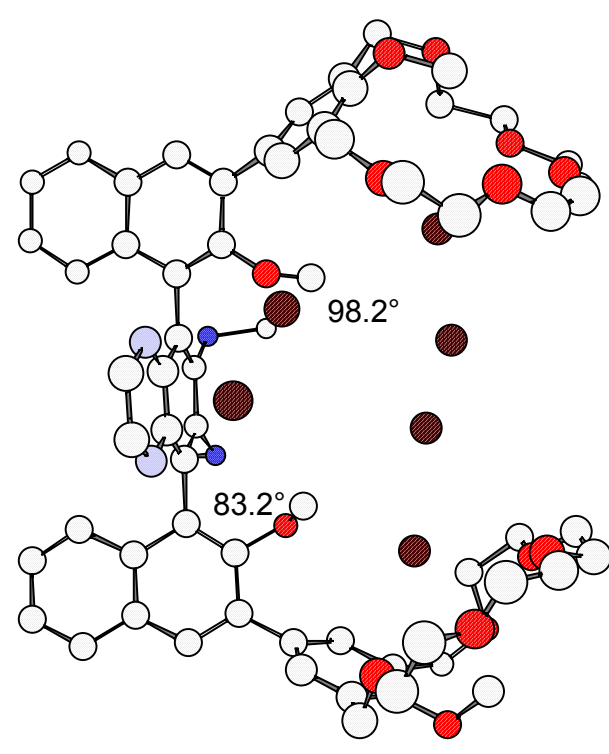

X-ray structure of $13 \cdot 6 \mathrm{H}_{2} \mathrm{O}$

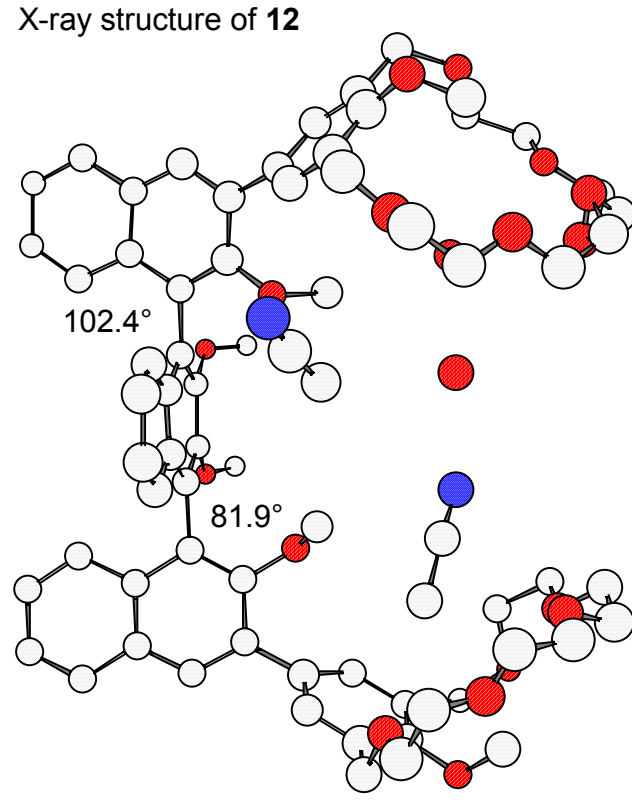

X-ray structure of $13 \cdot 2 \mathrm{H}_{2} \mathrm{O} \cdot 2 \mathrm{CH}_{3} \mathrm{CN}$

Tetrahedron. 2002, 58, 5611-5617 
Figure S-3. X-Ray Structures of the Quaternaphthalene

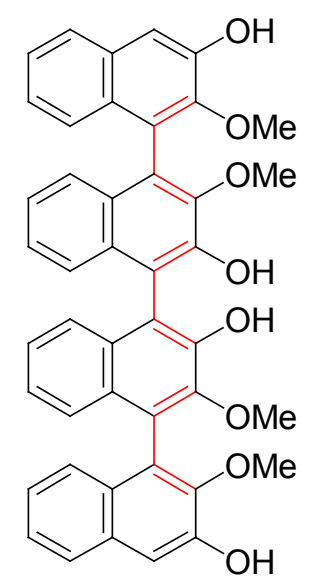

14

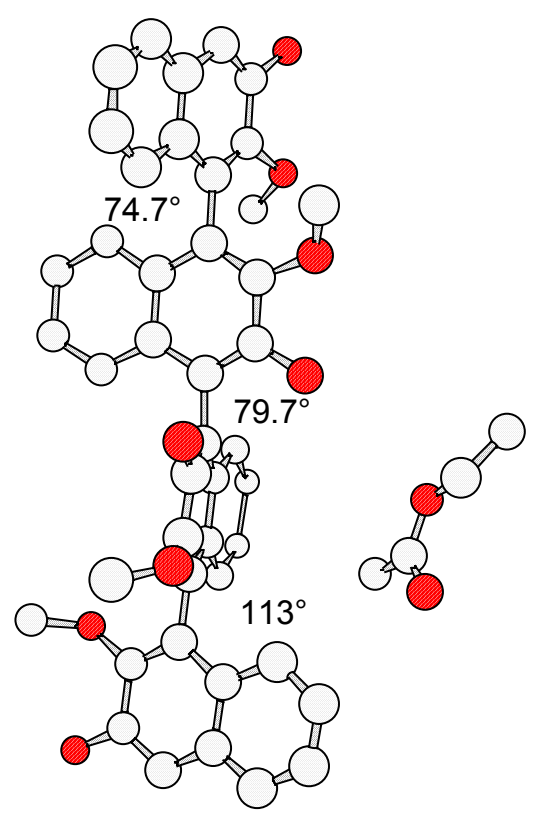

Tetrahedron: Asymmetry. 1996, 7, 2199-2202 
Figure S-4. Lowest-energy structure (calculated with CONFLEX-MM2) of $(S, S, S, S, S, S, S)-\mathbf{3 a}$.
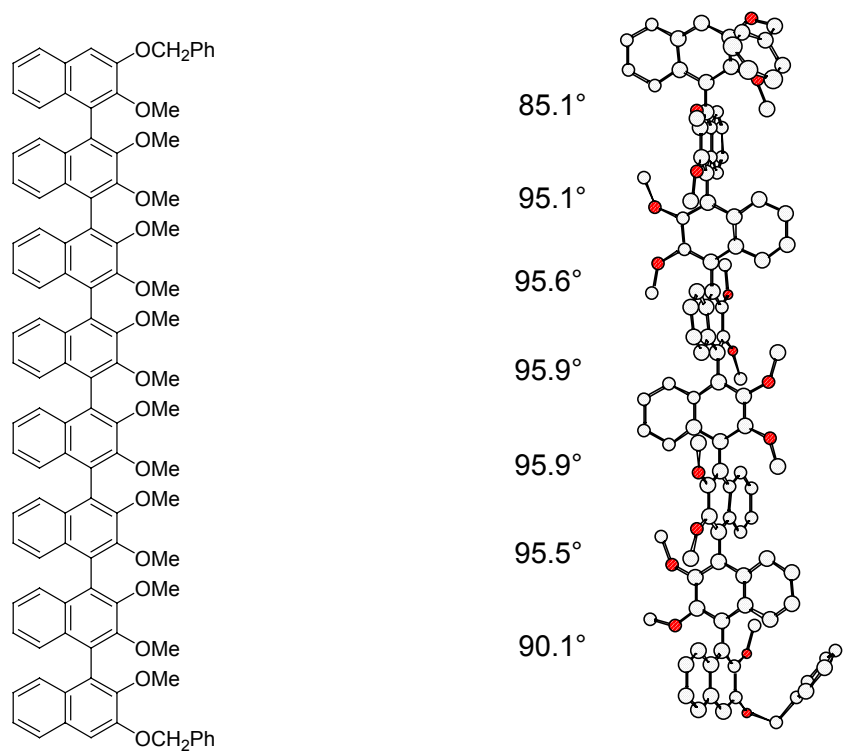

$(S, S, S, S, S, S, S)$

Figure S-5. Lowest-energy structure (calculated with CONFLEX-MM2) of $(S, S, S, R, S, S, S)-\mathbf{3 a}$.
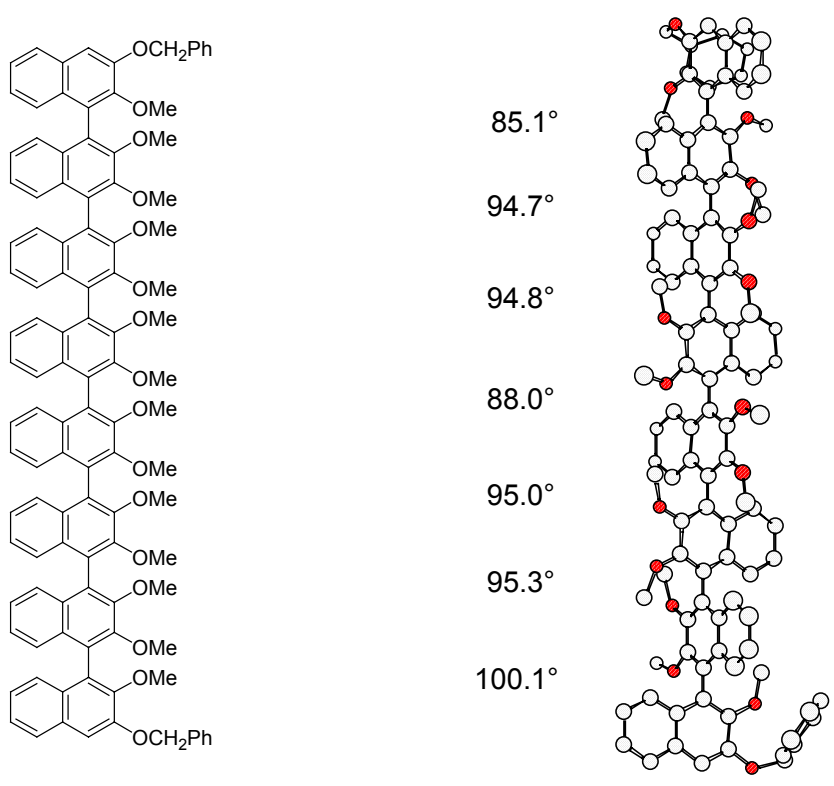

$(S, S, S, R, S, S, S)$ 
Figure S-6. Lowest-energy structure (calculated with CONFLEX-MM2) of $(S, S, S, S, S, S, S)$-7a (dimethylamide form).
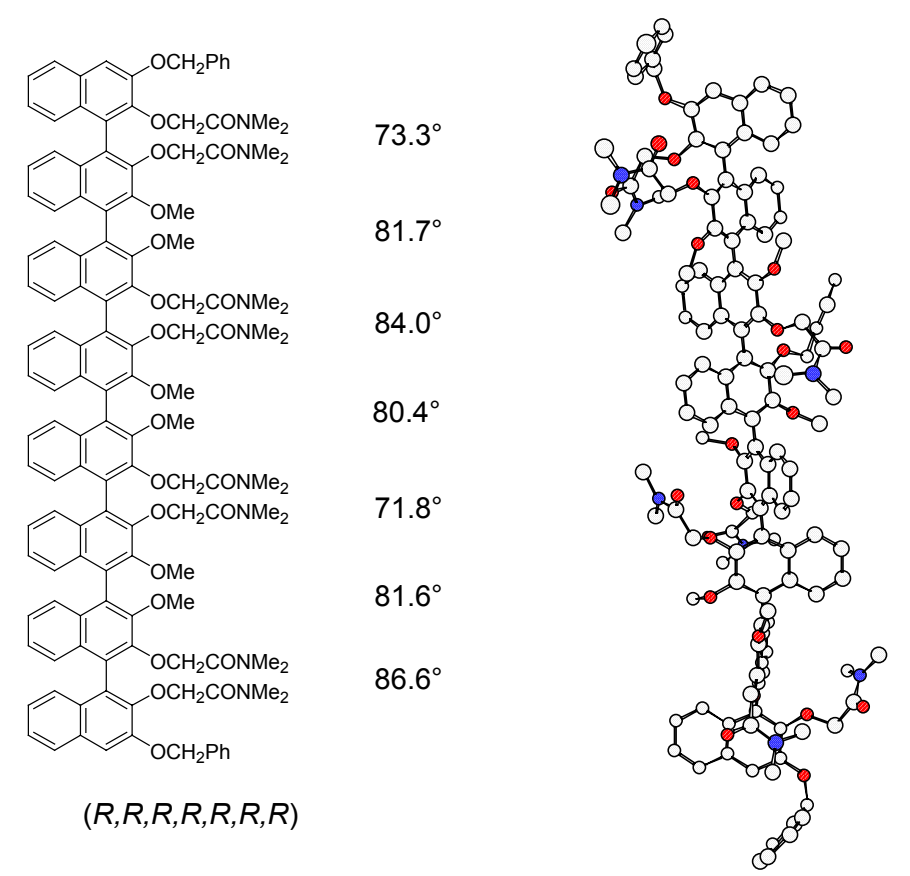

Figure S-7. Lowest-energy structure (calculated with CONFLEX-MM2) of $(S, S, S, S, S, S, S)-\mathbf{3 b}$.
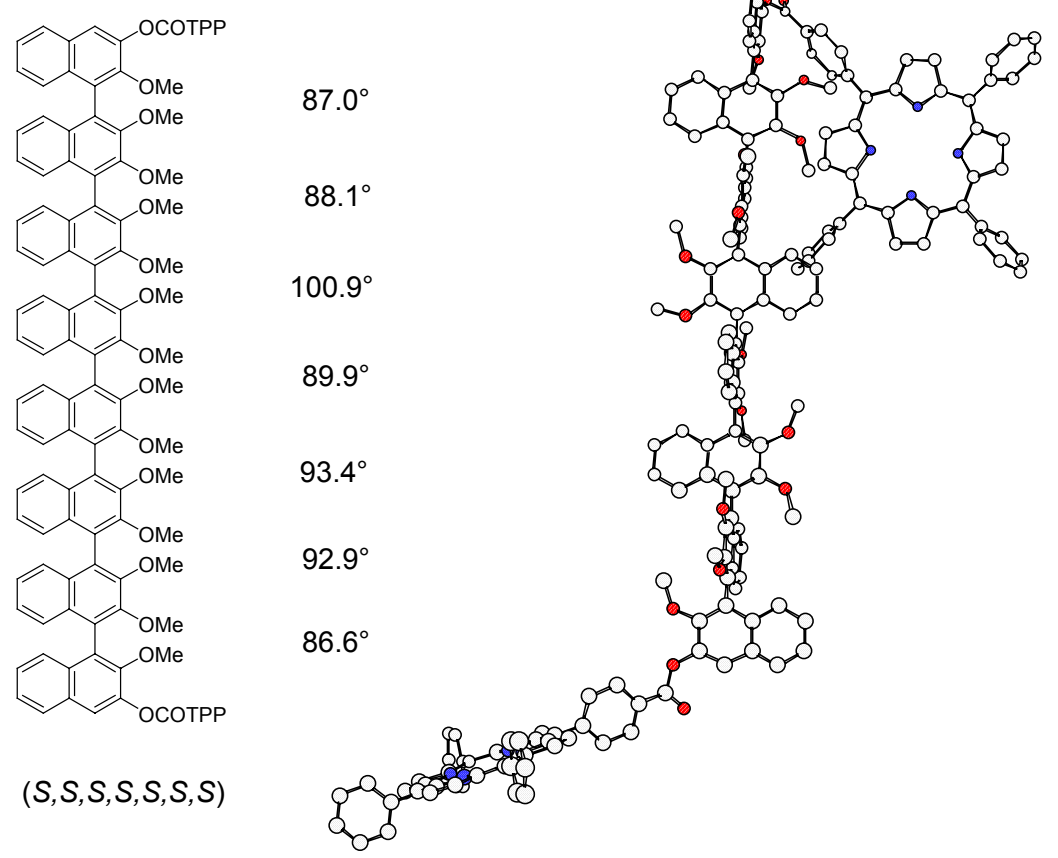
Figure S-8. Lowest-energy structure (calculated with CONFLEX-MM2) of (S)-1b.

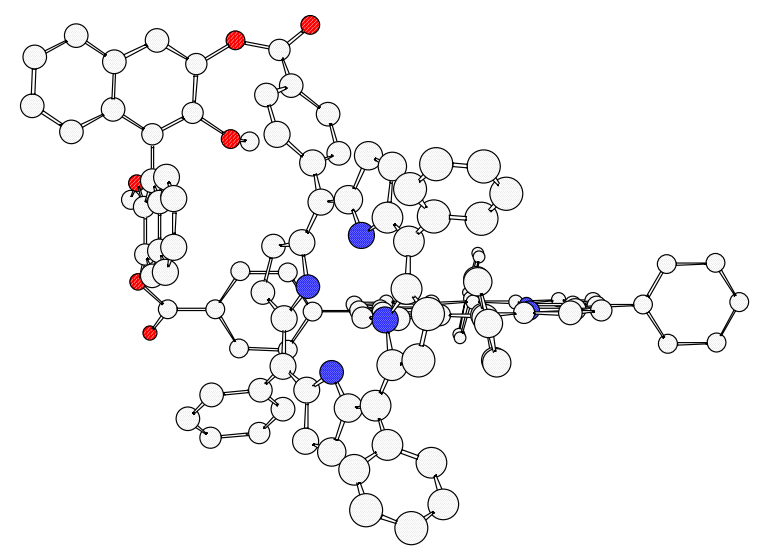

Side View

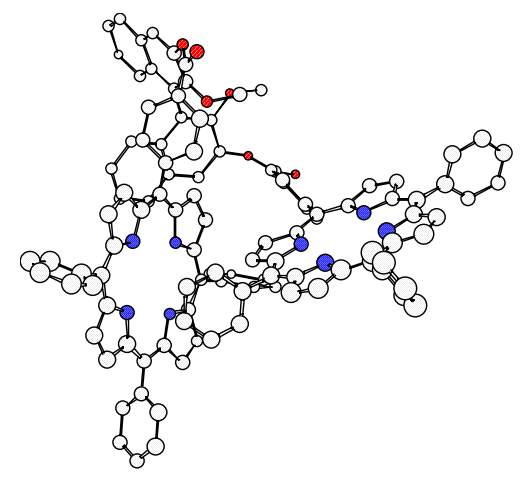

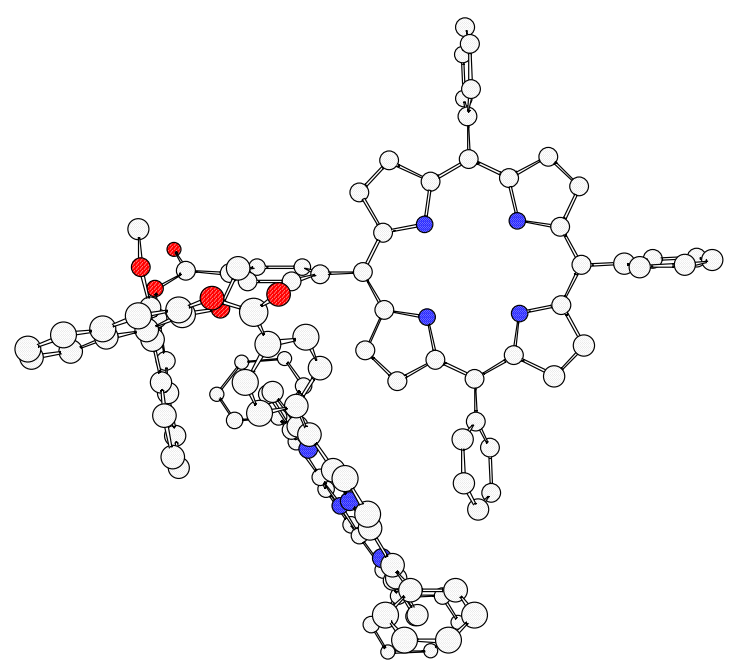

Top View

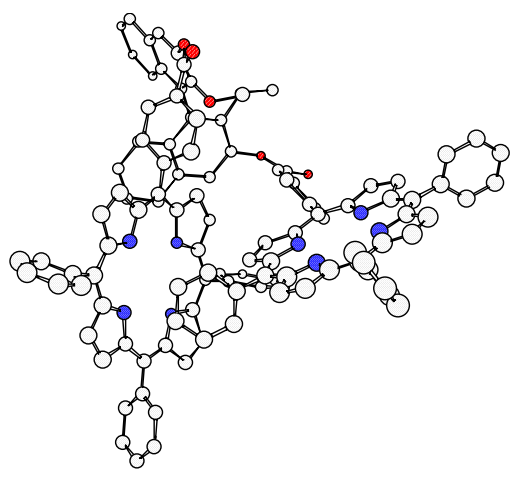

Stereo View 
Figure S-9. Original (before smoothing) CD spectra of $\mathbf{1 b}-\mathbf{4 b}$.

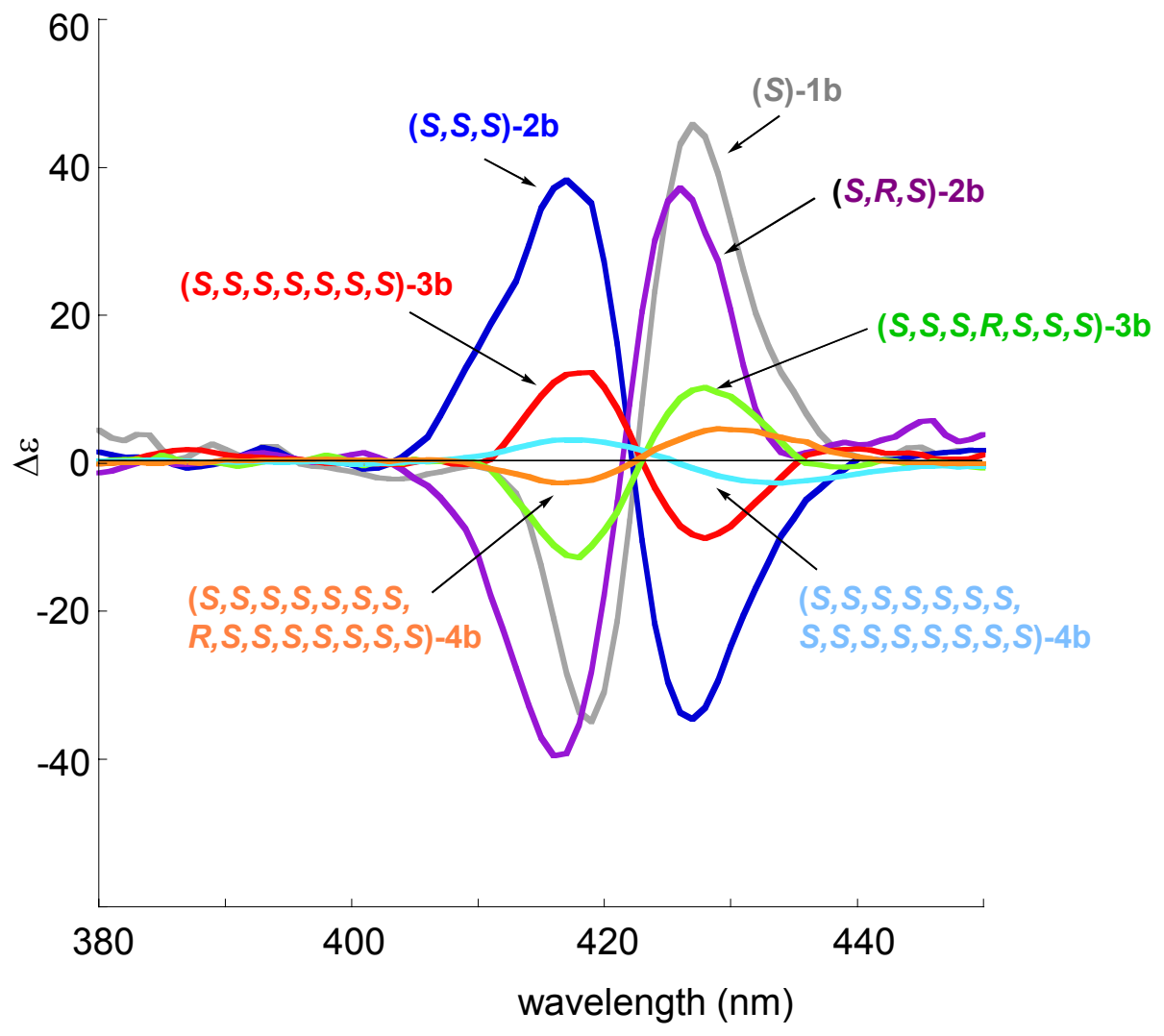

Conditions: JASCO J-720W; solvent, $\mathrm{CHCl}_{3}$; light path length, $1.0 \mathrm{~mm}$; Temp, $25{ }^{\circ} \mathrm{C}$; Scanning Speed, $50 \mathrm{~nm} / \mathrm{min}$; Data Pitch, $1 \mathrm{~nm}$; Accumulations, 16 (for 1b-3b) and 100 (for 4b). 
Figure S-10. Original (before smoothing) CD spectra of $\mathbf{5 b} \mathbf{b} \mathbf{7 b}$.

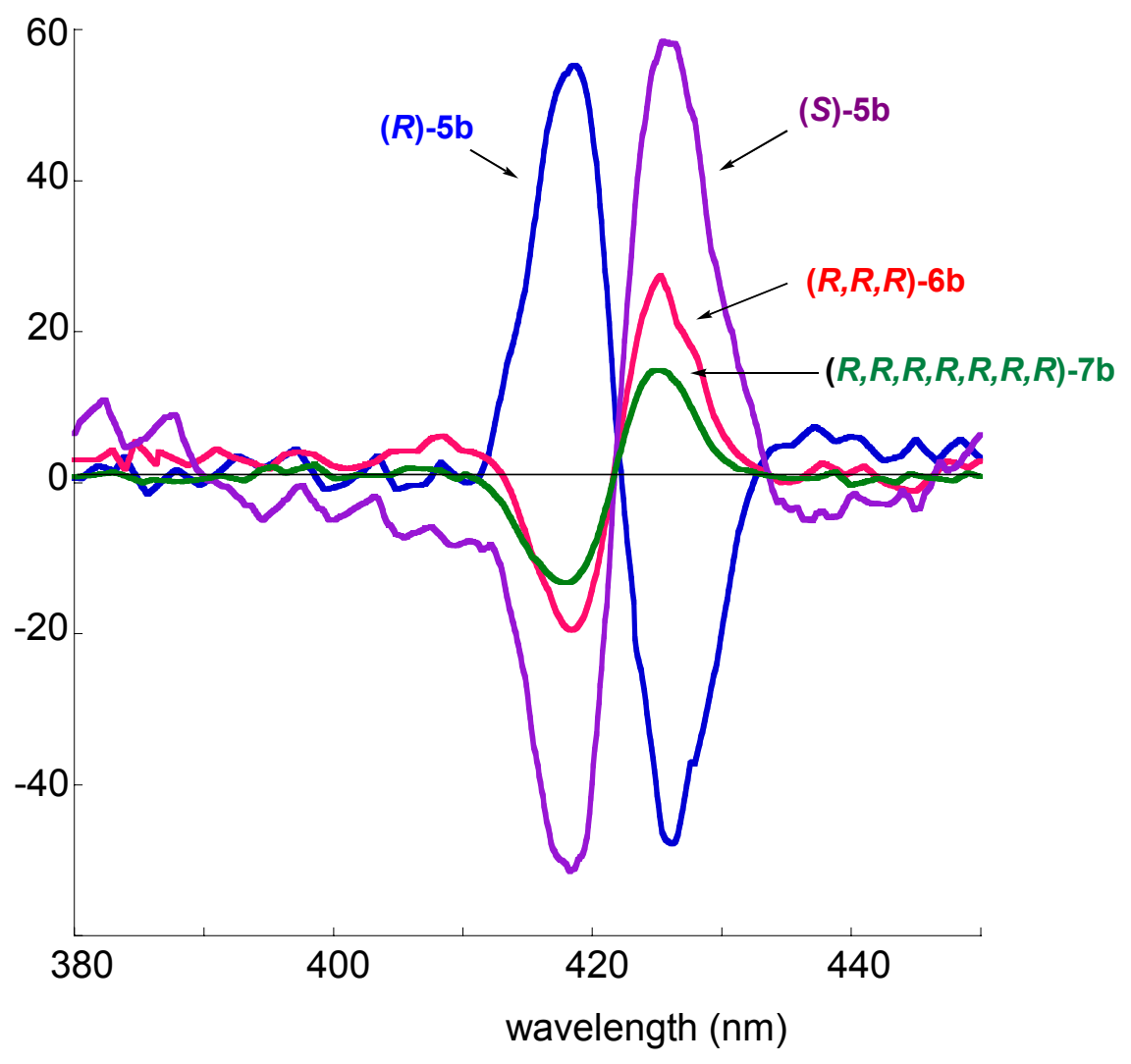

Conditions: JASCO J-720W; solvent, $\mathrm{CHCl}_{3}$; light path length, $1.0 \mathrm{~mm}$; Temp, $25{ }^{\circ} \mathrm{C}$; Scanning Speed, 50 nm/min; Data Pitch, $0.1 \mathrm{~nm}$; Accumulations, 4. 
Figure S-11. Original (before smoothing) CD spectra of 9b-11b.

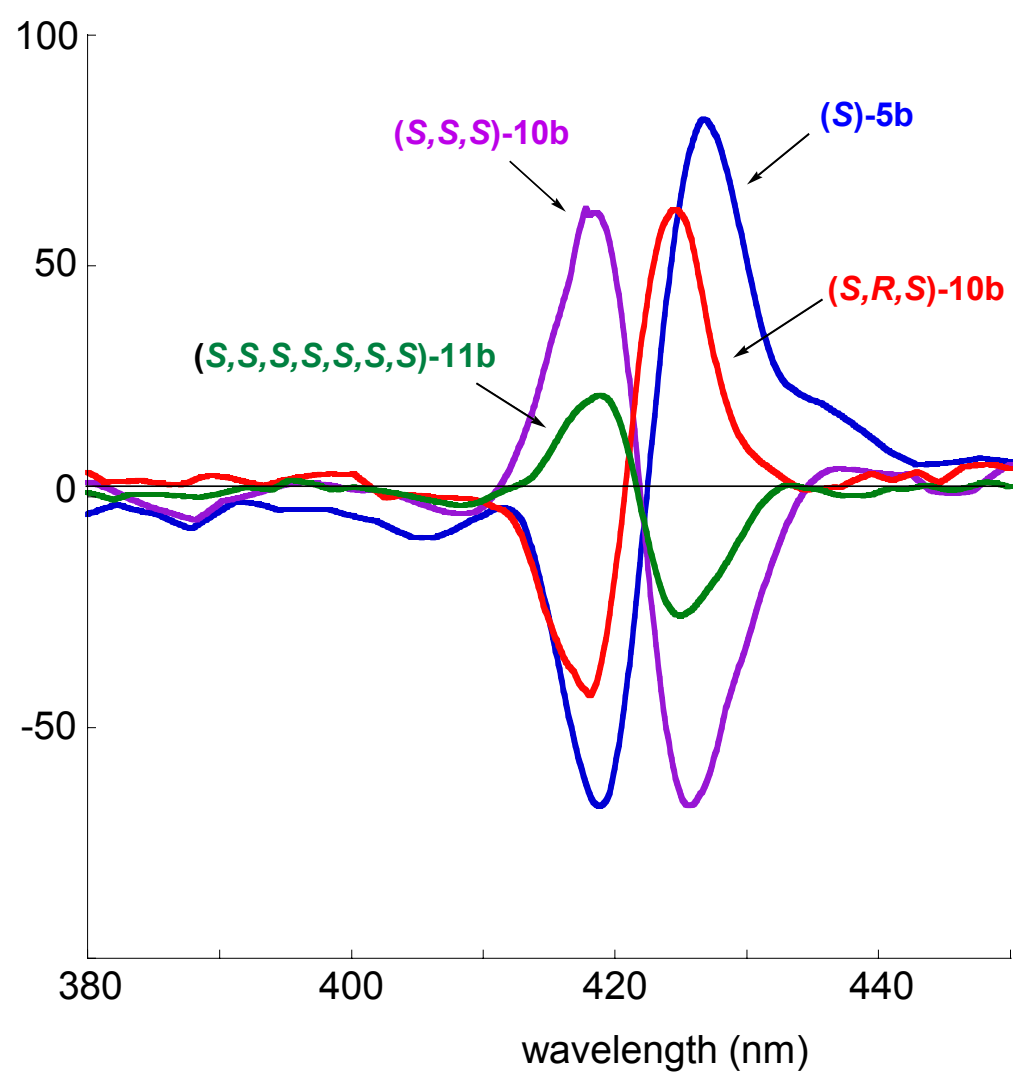

Conditions: JASCO J-720W; solvent, $\mathrm{CHCl}_{3}$; light path length, $1.0 \mathrm{~mm}$; Temp, $25{ }^{\circ} \mathrm{C}$; Scanning Speed, $50 \mathrm{~nm} / \mathrm{min}$; Data Pitch, $0.1 \mathrm{~nm}$; Accumulations, 5. 